\title{
Factores que determinan el desempeño educativo en el Uruguay, 2003-2006
}

\author{
Cecilia Oreiro y Juan Pablo Valenzuela
}

RESUMEN

Se analiza el desempeño de la Educación Media en el Uruguay sobre la base de la prueba del Programa para la Evaluación Internacional de Alumnos (PISA), Matemática 2003 y 2006. Se procura investigar las diferencias en la distribución de puntajes, identificar las variables que repercuten en el desempeño de los alumnos y analizar su evolución e importancia entre ambos años. Para ello se define una función de producción de resultados educativos y se aplican diferentes metodologías de descomposición. Se concluye que el bajo incremento en el puntaje entre 2003 y 2006 oculta movimientos internos que obedecen principalmente a un aumento generalizado en la eficiencia del sistema escolar, sobre todo en los establecimientos públicos, pero que en parte es neutralizado por la menor dotación de recursos y, en particular, por las desfavorables condiciones socioeconómicas y culturales de las familias de los estudiantes. Asimismo, los cambios son en su mayoría redistributivos. privadas, Uruguay coreiro@iecon.ccee.edu.uy

Juan Pablo Valenzuela es investigador del Centro de Investigación Avanzada en Educación (ciae) y del Departamento de Economía de la Universidad de Chile.jp.valenzuelab@gmail.com 


\section{I}

\section{Introducción}

El objetivo del presente trabajo es analizar la situación del Uruguay en materia de Educación Media, e identificar los factores que explican las diferencias en los resultados obtenidos por los estudiantes en la prueba del Programa para la Evaluación Internacional de Alumnos (PISA, por sus siglas en inglés) en los años 2003 y 2006, específicamente en la disciplina de Matemática.

La prueba PISA ha sido aplicada desde el año 2000 en los países miembros de la Organización de Cooperación y Desarrollo Económicos (OCDE) y en un grupo de países socios, a los estudiantes de 15 años independientemente del curso en que se encuentren. Estos datos permiten examinar cómo varía el desempeño de los alumnos según el grado que cursan, lo que constituye un indicador de cuánto van aprendiendo a medida que avanzan.

El análisis se centra específicamente en la prueba de Matemática, dado que en 2003 el foco principal de la evaluación estuvo en esta asignatura, que es la única que permite comparar los resultados con los de 2006. El propósito de la investigación es determinar si existen diferencias relevantes entre los puntajes PISA 2003 y 2006, identificar factores (características de los alumnos, de los centros educativos o institucionales) que explican el diferencial de puntajes PISA entre ambos años, y estimar si las diferencias obedecen a variaciones

$\square$ Este trabajo está basado en la Tesis final para la Maestría en Economía de la Facultad de Economía y Negocios de la Universidad de Chile, enero 2011. Los autores agradecen la valiosa colaboración de Alejandro Sevilla. en la magnitud de dichos factores, en la "eficiencia" en su uso o en ambas.

De acuerdo con Valenzuela y otros (2009a), para realizar el trabajo se utilizan metodologías de descomposición de diferencias de resultados como las de Oaxaca (1973), Blinder (1973) y Juhn, Murphy y Pierce (1993). Asimismo, se realiza un análisis de microsimulaciones en consonancia con Bourguignon, Fournier y Gurgand (1998).

La importancia de esta investigación, como contribución al fomento del desarrollo económico y social de los países latinoamericanos, reside en la posibilidad de extraer conclusiones sobre la calidad del sistema educativo y su heterogeneidad, permitiendo identificar desafíos para su mejoramiento. La prueba PISA es una herramienta que hace posible comparar los avances con los de los países desarrollados del mundo, como también entre las economías de la región y otros países de similar nivel de desarrollo. Asimismo, el empleo de técnicas de descomposición poco difundidas para el área de la educación se traduce en un avance metodológico de gran utilidad para entender mejor la evolución de los cambios en los resultados educativos, que puede ser replicado para otros sistemas escolares de la región así como con el objetivo de realizar comparaciones entre países.

El artículo se estructura de la siguiente manera: en la sección II que sigue a la Introducción se establece el marco de referencia. Luego, en la sección III se plantea la metodología de trabajo. En la sección IV se exponen los resultados y en la sección V se hace una comparación de estos. Finalmente, en la sección VI se entregan las conclusiones. 


\section{II}

Marco

\section{Contexto socioeconómico}

El Uruguay se ha situado históricamente entre los países latinoamericanos que presentan menores niveles de desigualdad e incidencia de la pobreza. El ingreso per cápita crece hasta mediados de la década de 1990, su distribución permanece relativamente estable y la pobreza tiene una tendencia decreciente (Amarante y Perazzo, 2008). En la segunda mitad del decenio el ingreso comienza una fase descendente y su concentración aumenta levemente, mientras que la pobreza inicia una etapa de crecimiento. En 1999 empieza a gestarse una grave recesión económica que culmina con una profunda crisis en 2002. Todas las variables económicas empeoran, hay una drástica caída del producto interno bruto (PIB) y del ingreso per cápita, se agudizan los niveles de desigualdad y la pobreza registra un incremento muy pronunciado.

Durante el desarrollo de la crisis la emigración internacional se convirtió en la principal tendencia demográfica. Las estimaciones indican que el saldo migratorio negativo es de 100.000 personas en el período 2000-2004 y de 26.000 entre 2005 y 2006 (Pellegrino y Koolhaas, 2008). En cuanto a las características de la población uruguaya que emigra, en su mayoría suele ubicarse entre los 20 y 29 años, sobre todo hombres y con nivel educativo alto, superior en promedio a los residentes en el Uruguay. Además, se caracteriza por estar integrada en mayor medida por familias completas.

A partir de 2003, el nivel de actividad comienza a recuperarse y ese proceso se acelera entre 2004 y 2006, en que se observa un marcado crecimiento económico. Sin embargo, la recuperación económica no se hizo visible en la evolución de los ingresos de los hogares hasta fines de 2005, y recién en 2006 se detecta una reducción significativa de la indigencia y de la incidencia e intensidad de la pobreza (PNUD, 2008).

\section{Panorama educativo en el Uruguay}

Actualmente, en el Uruguay la educación básica obligatoria comprende nueve años: seis de primaria y tres de ciclo básico de enseñanza media, que se imparte en liceos y escuelas técnicas con el mismo currículo. Desde el punto de vista de su administración, los liceos dependen del Consejo de Educación Secundaria (CES) y las escuelas técnicas del Consejo de Educación TécnicoProfesional (CETP). El segundo ciclo de la enseñanza media consta de tres años y puede cursarse en liceos con la modalidad de Bachillerato Diversificado o en escuelas técnicas con la modalidad de Bachillerato Tecnológico. A su vez, el CETP ofrece cursos básicos de capacitación o de formación profesional básica, así como cursos de formación profesional superior.

Luego de la crisis de 2002, la recuperación económica se traduce en un leve traslado de la matrícula al sector privado, que aumenta su participación relativa en el nivel, aunque sin alterar la estructura tradicional de una oferta que continúa siendo predominantemente pública (Cardozo, 2008). La fase de expansión se interrumpe en 2004. La matrícula en la Educación Media cae en los años 2004 y 2005 y se mantiene estable en 2006. La caída en 2004 obedece a una disminución de la matrícula en los centros públicos, mientras que en la educación técnica y privada aumenta el número de estudiantes. En 2005 el descenso matricular se registra también a nivel de la educación técnica, mientras que el sector privado crece por segundo año consecutivo, pero el saldo total vuelve a ser negativo en ese año. La disminución total de la matrícula entre 2003 y 2005 se debe, por una parte, al descenso en los egresos de la educación primaria y, por otra, a los eventos de emigración internacional (ANEP, 2007a).

Si se considera, además, el número de personas que potencialmente deberían acceder a la educación con respecto al número de personas que efectivamente concurren a ella, se obtiene una medida más exacta del nivel de cobertura de la enseñanza en cada sector. La tasa de asistencia de las personas de 15 años a la Educación Media en 2006 es del 79,7\%, lo que implica un aumento de 5 puntos porcentuales en relación con la asistencia en 2003. Este incremento obedece a una mejora en la cobertura rural, que pasa del $51,8 \%$ al $69,2 \%$ en el período, mientras que la tasa de cobertura en las localidades urbanas con más de 5.000 habitantes se mantiene constante (ANEP, 2007b).

El Uruguay ha participado en las pruebas PISA 2003 y 2006, obteniendo desempeños entre los más altos de América Latina. Sin embargo, con respecto a los países de la OCDE los resultados son considerablemente inferiores. Asimismo, el desempeño alcanzado en el Uruguay se 
ubica entre los más heterogéneos de los participantes, mucho más desigual que el desempeño del conjunto de los países de la oCDE e incluso, en 2006, que el de los restantes países de la región (ANEP, 2004 y 2007b).

Entre 2003 y 2006 el promedio del Uruguay en la prueba PISA de Matemática aumenta de 422,2 a 426,8 puntos, con un incremento de tan solo un 1,1\%; asimismo, esta diferencia no es estadísticamente significativa. Sin embargo, cabe plantearse si lo acontecido con el puntaje promedio del Uruguay entre ambos períodos es el resultado de movimientos contrapuestos ocurridos en diferentes variables sociales e institucionales. La existencia de factores que repercuten con distinta dirección y magnitud se analizará aplicando metodologías de descomposición, a fin de descomponer el efecto de cada uno de ellos.

Además, la prueba PISA también presenta sus resultados mediante una clasificación ordinal del nivel de desempeño escolar. Para el caso de Matemática se identifican seis niveles de desempeño. En el gráfico 1 se presentan los porcentajes de estudiantes en cada uno de los niveles en 2003 y 2006. Como puede observarse, en 2003 el $49 \%$ de los estudiantes uruguayos se encuentran bajo el nivel 2, es decir, un nivel que no permite manejar las competencias mínimas de las matemáticas en la vida cotidiana, y al mismo tiempo corren un alto riesgo de quedar excluidos de la participación ciudadana y de los ámbitos laborales enmarcados por una sociedad de la información y del conocimiento. A su vez, el 48,5\% se hallan en niveles intermedios (2, 3 y 4), mientras que en los niveles superiores ( 5 y 6 ) apenas alcanzan a un $2,6 \%$; estos dos últimos niveles involucran un alto desarrollo de las competencias en relación con la edad de los estudiantes y constituyen el grupo en el que la labor del sistema educativo ha sido más destacada. La comparación de 2006 con respecto a 2003 indica una disminución de un $2,8 \%$ de estudiantes ubicados en los niveles más bajos de desempeño y un incremento de un $2,8 \%$ en los niveles intermedios, mientras que se mantiene casi inalterado el porcentaje de estudiantes en los niveles destacados. Esto estaría señalando un cambio con sentido progresivo, pero muy paulatino, que de mantenerse en el futuro podría requerir medio siglo para que ningún joven uruguayo se encuentre en los niveles críticos en el manejo de las matemáticas.

GRÁFICO 1

\section{Porcentaje de estudiantes por nivel de desempeño, PISA Matemática 2003 y 2006}
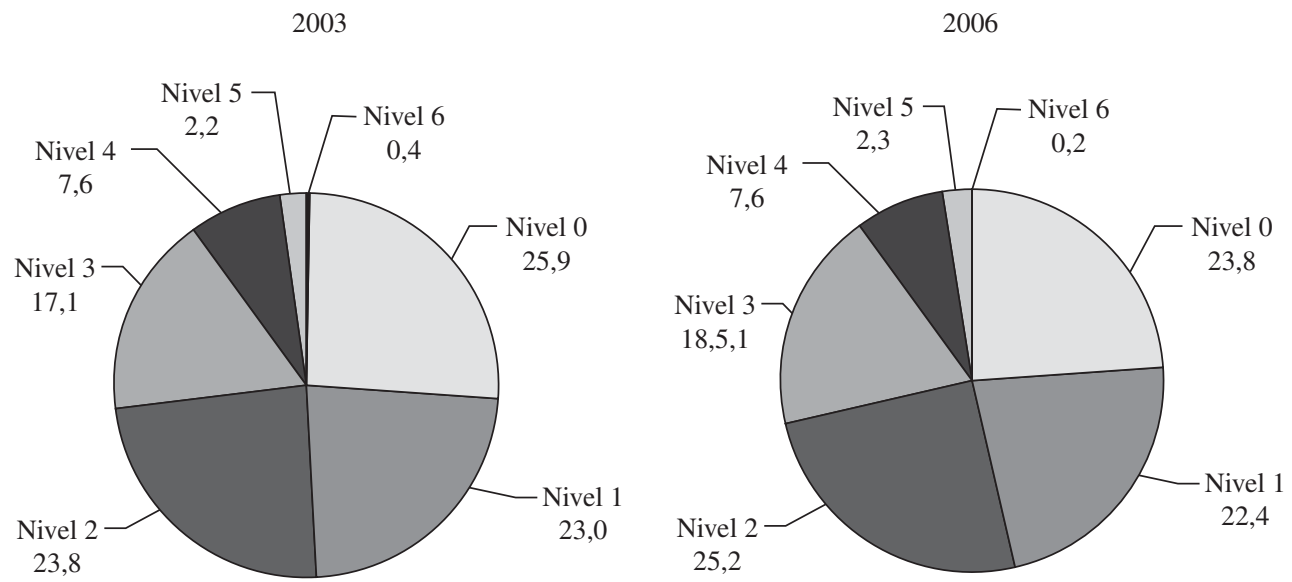

Fuente: elaboración propia sobre la base de datos de la Organización de Cooperación y Desarrollo Económicos (OCDE), PISA 2003 y PISA 2006 [en línea] http://www.pisa.oecd.org/document/51/0,3746,en_32252351_32235731_39732595_1_1_1_1,00.html 


\section{III}

\section{Metodología}

\section{Metodología general}

\section{a) Función de producción}

El primer paso para especificar la metodología es definir una función de producción que relacione el resultado en la prueba PISA de cada alumno con un conjunto de variables explicativas (variables socioeconómicas del alumno y su familia, así como variables del establecimiento educativo e institucionales):

$$
Y_{i}^{t}=X_{i}^{t} \beta^{t}+\varepsilon_{i}^{t}
$$

donde $Y_{i}^{t}$ indica el resultado obtenido por el alumno $i$, en un determinado centro educativo, en el momento $t ; X_{i}^{t}$ representa las características observables a nivel del alumno, del centro educativo o institucionales; $\beta^{t}$ corresponde a los coeficientes estimados para las distintas variables de control $^{1}$; $\varepsilon_{i}^{t}$ es el término de error, que se supone con distribución normal con media cero y desvío $\sigma_{\varepsilon}^{t}$, e independiente de las variables exógenas del modelo.

La selección de variables utilizadas para los análisis estadísticos se muestra en el cuadro A. 1 del anexo ${ }^{2}$, y en el cuadro A.2 se presentan las principales estadísticas descriptivas.

\section{Metodología de imputación}

Debido al alto porcentaje de datos faltantes en la prueba PISA $^{3}$, debe optarse por una forma de tratamiento de estas observaciones. Ammermüller (2004) afirma que comúnmente se elimina la observación de la regresión

\footnotetext{
${ }^{1}$ Dada la forma en que la prueba PISA expresa los resultados, los coeficientes son estimados trabajando con los 5 valores plausibles, lo que implica estimar las regresiones cinco veces y luego calcular el promedio de las estimaciones para hallar el valor del estadístico. La varianza, por su parte, es ajustada por cada estimación y en conjunto. ${ }^{2}$ La selección de variables está realizada sobre la base de varios trabajos precedentes a nivel nacional e internacional, en particular las investigaciones de Llambí y Perera (2008); Méndez y Zerpa (2009) y Hanushek y Woessmann (2010).

${ }^{3}$ En ambas pruebas los datos faltantes se concentran en las variables a nivel del centro educativo, con un máximo de un $4 \%$ de faltantes para el año 2003 en la variable "Porcentaje de profesores titulados" y un máximo de un 3,8\% de faltantes para el año 2006 en la variable "Escasez de profesores de Matemática".
}

cuando falta algún valor de alguna variable explicativa. Esto conduce a una gran reducción del número de observaciones que pueden utilizarse en las estimaciones ${ }^{4}$. Asimismo, lleva a un sesgo de selección en la muestra si los valores no resultan aleatoriamente faltantes.

De acuerdo con Valenzuela y otros (2009a), el método aplicado en el presente trabajo consiste en la imputación del valor de la mediana de un subgrupo similar, es decir, con similares valores en las distintas variables de control, de tal forma que las observaciones con datos imputados pertenezcan a subgrupos relativamente homogéneos. Los grupos de control para definir los subgrupos se van reduciendo de modo de alcanzar progresivamente la imputación para todas las observaciones con datos faltantes ${ }^{5}$.

El hecho de trabajar con datos que presentan variabilidad, ya sea en el puntaje de cada caso o en valores imputados para datos faltantes, hace que aplicar simplemente los valores promedio pueda implicar la omisión de la incertidumbre con que se están midiendo. Esto puede traducirse en una distorsión en la estimación de los errores estándar calculados para los parámetros, lo que es sobre todo relevante para aquellos efectos que se encuentran en el límite de la significancia. En este sentido, el cálculo de los errores estándar incluye los pesos de las 80 réplicas repetidas equilibradas (BRR, por sus siglas en inglés) proporcionadas en la base de datos, con la corrección de Fay $(0,5)$. Estos errores estándar proporcionan el grado de variación de cualquier estadístico con respecto al parámetro poblacional, y las réplicas consideran el diseño complejo de la encuesta, realizado en dos etapas con un diseño de muestra estratificado.

\section{Metodología de descomposición}

La estrategia metodológica aplicada en este trabajo consiste en distintas técnicas de descomposición de

\footnotetext{
${ }^{4}$ En especial, si se realizaran las estimaciones sin corregir por datos faltantes, se perderían 461 observaciones en la muestra de 2003 y 377 en la muestra de 2006, es decir, cerca de un $8 \%$ en ambos casos.

${ }^{5} \mathrm{La}$ efectividad del método, testeado como el porcentaje de aciertos entre la variable observada y la variable imputada por cada iteración, alcanza al $60 \%$, lo que supera las metodologías aplicadas en los trabajos de Ammermüller (2004) y Fuchs y Woessmann (2004) .
} 
diferencias de resultados: Oaxaca (1973); Blinder (1973); Juhn, Murphy y Pierce (1993); y Bourguignon, Fournier y Gurgand (1998).

Las dos primeras metodologías fueron aplicadas en los trabajos de Valenzuela y otros (2009b) y Bellei y otros (2009) para identificar factores que explican las diferencias de resultados obtenidos por los estudiantes chilenos en Matemática y Lenguaje en la prueba PISA 2006, respecto de los obtenidos por los estudiantes de Polonia, España y el Uruguay. Asimismo, en Valenzuela y otros (2009a) se aplica, además, la metodología de microsimulaciones para identificar los factores explicativos del mejoramiento de resultados en la prueba PISA de Lectura de los alumnos chilenos de 15 años entre 2001 y 2006 , e identificar factores que contribuyen a explicar el aumento de la desigualdad en dichos resultados. Estas fuentes constituyen la principal referencia metodológica utilizada en el presente trabajo.

a) Descomposición de Oaxaca (1973) y Blinder (1973)

El método de descomposición propuesto por Oaxaca (1973) y Blinder (1973) permite descomponer el efecto de los diferenciales de resultados entre dos grupos de individuos o entre dos años considerados. En esta metodología la descomposición se realiza en tres efectos, uno de ellos se corresponde con los diferentes resultados que pueden percibir los individuos pertenecientes a un mismo grupo como consecuencia de sus diferentes características: "efecto características". El segundo se corresponde con la diferencia en la eficiencia en el uso de estas características entre los grupos: "efecto retorno". Y, por último, el "efecto interacción" capta el efecto combinado de las diferencias de características y retorno.

Dados los dos años considerados (sean $t$ y $t^{\prime}$ ), una variable de resultados promedio en Matemática $(Y)$ y un conjunto de variables explicativas, la técnica de OaxacaBlinder permite estimar cuánto de la diferencia en las medias de resultados se explica por diferencias en las variables explicativas de cada año

$$
R=E\left(Y_{t}\right)-E\left(Y_{t}^{\prime}\right)
$$

donde $E(Y)$ indica el valor esperado de los resultados en Matemática en la prueba PISA en un año particular.

Obteniendo separadamente para las dos muestras $\hat{\beta}_{t}$ y $\hat{\beta}_{t}$, los estimadores por mínimos cuadrados ordinarios (MCO) de $\beta_{t}^{\prime}$ y $\beta_{t}$ y utilizando $\bar{X}_{t}^{\prime}$ y $\bar{X}_{t}$ como los estimadores de $E\left(X_{t}^{\prime}\right)$ y $E\left(X_{t}\right)$, la descomposición puede expresarse como:

$$
\begin{array}{r}
\hat{R}=\bar{Y}_{t}-\bar{Y}_{t^{\prime}}=\left(\bar{X}_{t}-\bar{X}_{t^{\prime}}\right)^{\prime} \hat{\beta}_{t^{\prime}}+\bar{X}_{t^{\prime}}, \\
\left(\hat{\beta}_{t}-\hat{\beta}_{t^{\prime}}\right)+\left(\bar{X}_{t}-\bar{X}_{t^{\prime}}\right)^{\prime}\left(\hat{\beta}_{t}-\hat{\beta}_{t^{\prime}}\right)
\end{array}
$$

En esta ecuación, $\bar{Y}$ refleja el promedio de puntaje obtenido en Matemática para cada año de la prueba PISA. El primer término de la ecuación 3 corresponde al efecto de las medias de las variables de control, que son las variables explicativas incorporadas en la función de producción (véase la ecuación 1), es decir, variables a nivel del estudiante, del centro educativo y a nivel institucional. El segundo término de la ecuación 3 corresponde al efecto de las diferencias de los coeficientes asociados a estas variables observadas, es decir, la productividad o efectividad de estos factores. Por último, el tercer término refleja la interacción de ambos efectos.

b) Descomposición de Juhn, Murphy y Pierce (1993)

Juhn, Murphy y Pierce (1993) generalizan el enfoque de Oaxaca-Blinder (1973), desarrollando una metodología que permite descomponer los cambios en la distribución de los resultados, y ver su efecto en las diversas partes de la distribución.

La metodología propuesta requiere inicialmente obtener el residuo $\varepsilon_{i t}$ en función de dos elementos: el percentil que ocupa el individuo $i$ en el momento $t$ en la distribución del residuo, $\theta_{i t}$, y la función de distribución de los residuos de los resultados en $t, F_{t}(\cdot)$. Luego, por definición se tiene que:

$$
\varepsilon_{i t}=F_{t}^{-1}\left[\frac{\theta_{i t}}{X_{i t}}\right]
$$

De esta forma, es posible estimar la distribución de los resultados para cada año, separando los efectos provenientes de cambios en las características observables, sus retornos y los residuos, para lo que se realizan diversas estimaciones de resultados para cada año. Una primera estimación corresponde a la forma regular para cada año:

$$
R_{i t}^{(1)}=\beta_{t} X_{i t}+F_{t}^{-1}\left[\frac{\theta_{i t}}{X_{i t}}\right]
$$

Una segunda estimación corresponde a la estimación de resultados de un año $t$, considerando los retornos y residuos del otro año $t$ ':

$$
R_{i t}^{(2)}=\beta_{t}^{\prime} X_{i t}+F_{t}^{\prime-1}\left[\frac{\theta_{i t}}{X_{i t}}\right]
$$


Por último, se puede estimar de la forma regular para cada año, pero considerando únicamente los residuos del otro año:

$$
R_{i t}^{(3)}=\beta_{t} X_{i t}+F_{t}{ }^{(-1)}\left[\frac{\theta_{i t}}{X_{i t}}\right]
$$

Este método permite descomponer los cambios en la desigualdad de los resultados en tres componentes: "efecto características": $R_{t}^{(2)}-R_{i t}^{\prime((1))}$, "efecto retorno":

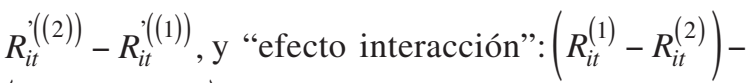

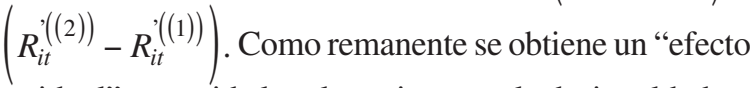
residual" que mide las alteraciones en la desigualdad no

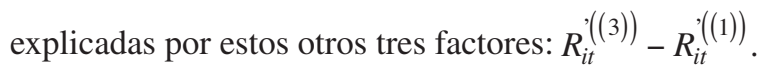

\section{c) Descomposición de Bourguignon, Fournier y Gurgand (1998)}

Otros autores generalizan el método de microsimulaciones, habilitando su utilización para comprender la evolución de la distribución total de resultados. La metodología fue originalmente desarrollada por Almeida dos Reis y Paes de Barros (1991) para un análisis de la desigualdad de los ingresos laborales. Posteriormente, fue generalizada para analizar la desigualdad de ingresos y la pobreza sobre la base del ingreso total per cápita de los hogares. El primer trabajo en esta dirección es el de Bourguignon, Fournier y Gurgand (1998), que aplica la metodología de descomposición del cambio en la desigualdad del ingreso de los hogares para la provincia china de Taiwán.

\section{Estimación de la función de producción y elec- ción del centro educativo}

El método de descomposición parte de la estimación de la función de producción (véase la ecuación 1). Esta función se estima para cada año y para cada tipo de establecimiento (público y privado) por MCO. Paralelamente se modela, para cada estudiante, la decisión de elección entre los dos tipos de establecimientos, utilizando una estimación logit.

Conviene hacer una advertencia con respecto al riesgo de sesgo de selección. Una posible relación positiva entre el tipo de centro en el que se estudia y los logros educativos puede estar producida por la existencia de un sesgo de selección. Este sesgo puede obedecer al hecho de que la elección del tipo de colegio por parte de las familias corresponde a una decisión endógena en función de sus propias características, lo que debe ser tenido en cuenta adecuadamente al plantear el análisis posterior ${ }^{6}$.

\section{Resultados contrafactuales por cambios en las características de los estudiantes}

En el marco de esta metodología, el cálculo del "efecto características" implica encontrar el resultado simulado de los individuos en el momento $t$ si, todo lo demás constante, una característica $k$ del vector $X_{k i}^{t}$ tiene la distribución del momento $t$ '. De acuerdo con Valenzuela y otros (2009a) se emplea una metodología distinta según el tipo de variables. Para el caso de las variables dicotómicas se considera el porcentaje no ponderado de los casos que cumplen con la característica en el período $t$ ' y ese dato se simula en $t$. Sin embargo, el que se cumpla la característica del período $t$ ' debe estar vinculado a cada individuo en $t$. Para observar la probabilidad vinculada a cada individuo de que cumpla con las características observadas se estima una regresión probit en t', obteniendo la probabilidad en $t$ de que cumpla con la característica, según el resto de las condiciones de $t$ '. Ordenando esta probabilidad en forma descendente se considera el punto de corte según el porcentaje observado de individuos que cumplen con la característica en $t^{\prime}$. Por otra parte, las variables categóricas son simuladas mediante la estimación de un multilogit en $t$ ' y nuevamente se asocia una probabilidad a cada individuo en $t$ de que cumpla con la característica, para luego hacer el punto de corte según el porcentaje observado de individuos que cumplen con esta característica en $t^{\prime}$.

En el caso de las variables continuas, esta estrategia implica considerar grupos poblacionales construidos a partir del tipo de establecimiento y tamaño de la loca$\operatorname{lidad}^{7}$. Sobre la base de estos grupos se considera el mínimo de observaciones coincidentes entre años para un mismo grupo, y con este conjunto de observaciones se construyen cuantiles de los que se obtiene la media por grupo y cuantil. A su vez, con esta media para cada año de la variable a simularse se construye un factor -relación entre la media del año $t$ ' y la media del año $t$ - que multiplica a la variable simulada por grupo subpoblacional y cuantil.

\footnotetext{
6 Una posible estrategia para lidiar con el sesgo de selección es el ajuste de Heckman en dos etapas; sin embargo, para su aplicación es necesario que el modelo de selección contenga al menos una variable de exclusión, lo que no fue posible encontrar en el presente trabajo. ${ }^{7}$ En algunos casos es necesario realizar ajustes para una simulación más precisa, por lo que los grupos poblacionales pueden considerar otras variables como, por ejemplo, grado o retraso.
} 


\section{Resultados contrafactuales por cambios en los retornos}

El proceso de microsimulación de los resultados educativos por cambios en el vector de retornos (vector de coeficientes de efectividad de insumos) implica determinar los resultados contrafactuales que obtendrían los estudiantes en el momento $t$ si, ceteris paribus, cambian los retornos de ciertas características, es decir, son los que corresponden al período $t$ '. En definitiva, este procedimiento implica simular el resultado de los estudiantes en el momento $t$ incorporando los parámetros estimados correspondientes a estos retornos para el período $t^{\prime}\left(\hat{\beta}^{t^{\prime}}\right)$, manteniendo las características observables y no observables y la estructura de selección de establecimientos prevalecientes en el momento $t$.

\section{Resultados contrafactuales por cambios en la selección de tipo de establecimiento}

El "efecto selección de establecimiento" o "efecto choice" representa el cambio en la distribución de resultados de los estudiantes del momento $t$, si se mantiene la estructura de selección del período $t$ ', dadas las demás condiciones correspondientes al período $t$, es decir, las características observables y no observables y los retornos a estas características. Para ello se estima una función logit para cada año, donde el valor 1 (uno) es asignado al caso en que el estudiante está matriculado en un establecimiento público.

La simulación corresponde a la obtención de una nueva elección de establecimiento, al incorporar los parámetros estimados $\left(\hat{\lambda}_{j}^{t^{\prime}}\right)$ para el período $t^{\prime}$. Con respecto al término de error de esta ecuación, el procedimiento consiste en calcular un residuo como el valor de la decisión observada (1 para matrícula en establecimientos públicos y 0 en establecimientos privados), menos la probabilidad determinada por la estimación logit. Se considera que la familia prefiere los establecimientos públicos siempre y cuando la probabilidad estimada con esta simulación sea igual o mayor que 0,5 ; para valores inferiores, se asume la elección de un establecimiento privado. Así, se simula una estructura de elección de establecimientos para los individuos del período $t$, si los parámetros representativos de la estructura de selección son los correspondientes al período $t^{\prime}$, dado todo lo demás referido al período $t$.

Al realizar esta simulación, los individuos del período $t$ pueden cambiar su elección de tipo de establecimiento con respecto a la que realmente tienen. Luego de simular la elección de establecimiento de los individuos en el período $t$ se les imputa el desempeño correspondiente al estado simulado. En aquellos casos en que el resultado de la simulación cambia la elección de establecimiento, no se tiene el término de error estimado de la función de producción, por lo que se seleccionan, de una distribución normal, términos aleatorios para estos residuos que cumplan con las decisiones del tipo de establecimiento efectivamente observado.

\section{a) Factores complementarios}

Entre los factores complementarios a la microsimulación se incluye, en primer lugar, la simulación de las variables no observables. Para ello se consideran subgrupos por tipo de institución y tamaño de la localidad. Se calcula un factor como la fracción entre el desvío estándar del residuo para cada uno de los años por grupo, y luego este factor es multiplicado por los residuos del año 2003 por subgrupo. Este procedimiento se realiza para los residuos de las dos funciones de producción por tipo de institución.

En segundo lugar, para el ajuste de los factores de expansión se calcula una fracción que refleja la relación entre la población que representa la muestra en el año 2006 de un subgrupo $m$ (según tipo de institución, tamaño de la localidad, grado y, para el caso de los centros públicos, también retraso), es decir, la suma de los factores de expansión para cada subgrupo respecto de la población representada en el año 2003 para el mismo subgrupo. El factor de expansión de cada observación del subgrupo $m$ del año 2003 se multiplica entonces por dicha fracción. 


\section{IV}

\section{Resultados}

\section{Resultados de la descomposición de Oaxaca- Blinder (1973)}

Para los efectos de la presente investigación, la metodología de Oaxaca-Blinder permite desagregar el cambio total en el puntaje correspondiente al período 2003-2006, entre el efecto de un cambio en las características y un cambio en el retorno de estas. El cambio total entre los dos años considerados es de 4,6 puntos, que se debe mayoritariamente al efecto retornos que alcanza a 11,2 puntos (véase el cuadro 1) y cuya magnitud es relevante, además de ser estadísticamente significativa, pues es bastante similar al mejoramiento que conllevaría el incremento en una desviación estándar del nivel socioeconómico y cultural de las familias. Esto implica que la eficiencia de las características en cuanto a resultados educativos es mayor en 2006 que en 2003. Por su parte, el efecto características es negativo, lo que implica que las características en el año 2006 resultan más desventajosas que en 2003. El efecto interacción es el menor de todos y con signo negativo.

Al separar los efectos entre los tres grupos de variables explicativas y las características de los estudiantes y de los centros educativos e institucionales, estos efectos se pueden analizar de manera más detallada. Los cambios negativos vinculados a la caída de las características se concentran principalmente en las variables relativas al estudiante. Por una parte, el índice socioeconómico y cultural (ISEC) explica la caída de 1,8 puntos en el promedio. Este índice es construido por el programa PISA a partir de variables relacionadas con el contexto familiar y su media pasa de $-0,35$ a $-0,51$ en el período (véase el cuadro A.2 del anexo). Si bien este comportamiento puede resultar llamativo en un contexto de recuperación económica, es posible plantearse varias hipótesis que lo explican. Por una parte, podría estar influido por la evolución de la matrícula en el período, que estaría provocando un traslado de aquellos estudiantes con mayor nivel socioeconómico desde el sector público al sector privado, y contribuyendo a la reducción promedio del ISEC en los centros públicos. Asimismo, se produce un aumento en la tasa de cobertura de la Educación Media debido sobre todo al incremento en la asistencia en las localidades más pequeñas. Este fenómeno significa retener en los centros educativos a un segmento de la población estudiantil que antes abandonaba la educación a una edad más temprana y que, probablemente, presenta indicadores socioeconómicos desfavorables, por lo que este hecho también podría explicar la evolución del ISEC en el período. Una última explicación se refiere a la importante emigración experimentada por el país en esos años, que protagonizaron los sectores socioeconómicos medios y medio-altos, factor que también podría estar influyendo en la evolución del ISEC.

Por otra parte, la evolución en el porcentaje de estudiantes que cursan tercero, cuarto y quinto grados, explica la reducción en el promedio de 0,4, 0,5 y 0,7, respectivamente. Otra razón que estaría causando esta caída en el puntaje promedio explicada por el efecto características, corresponde al aumento en el porcentaje de estudiantes que presentan retraso escolar. La única variable que registra un efecto positivo, aunque no significativo, es el sexo.

Al considerar las variables vinculadas al centro educativo, se observa que la disminución en el efecto par explica una reducción de 3 puntos en el efecto características, mientras que la caída en el tamaño promedio del colegio solo explica 0,8 puntos de este efecto. Por su parte, la aminoración del indicador de escasez de materiales educativos y el aumento del porcentaje de profesores titulados explican un cambio positivo en 1,3 y 1,5 puntos, respectivamente. El total de las variables relativas al centro educativo presenta un cambio con signo negativo aunque no significativo. En cuanto a las variables institucionales, ninguna de ellas resulta significativa, presentando la variable ficticia (dummy) correspondiente a selectividad signo negativo y la condición de colegio privado signo positivo.

Con respecto a los efectos vinculados a cambios en la eficiencia de las características (efecto retorno), destaca el incremento de la constante en 43,3 puntos, lo que se explica por una recomposición del efecto sumatorio de cada año adicional de educación — mejorando considerablemente aquellos con menor escolaridad-y por un aumento generalizado del sistema escolar entre todos los estudiantes uruguayos. Al contrario, durante el período se acrecienta la brecha de aprendizaje entre los estudiantes que presentan retraso escolar y los que no lo presentan, brecha que se traduce en un efecto negativo de 4,3 puntos.

En lo que se refiere a los retornos de las características del centro, el cambio total se explica básicamente por 
CUADRO 1

\section{Descomposición de Oaxaca-Blinder}

\begin{tabular}{|c|c|c|c|}
\hline Variables estudiante & Características & Retornos & Interacción \\
\hline Sexo $($ mujer $=1)$ & $\begin{array}{c}0,01 \\
(0,30)\end{array}$ & $\begin{array}{l}-3,02 \\
(2,24)\end{array}$ & $\begin{array}{c}0,00 \\
(0,11)\end{array}$ \\
\hline Tercer grado & $\begin{array}{l}-0,40 \\
(0,70)\end{array}$ & $\begin{array}{c}-1,53 \\
(1,28)\end{array}$ & $\begin{array}{c}0,08 \\
(0,15)\end{array}$ \\
\hline Cuarto grado & $\begin{array}{c}-0,49 \\
(2,35)\end{array}$ & $\begin{array}{r}-12,96 \\
(9,09)\end{array}$ & $\begin{array}{c}0,10 \\
(0,52)\end{array}$ \\
\hline Quinto grado & $\begin{array}{c}-0,69 \\
(1,60)\end{array}$ & $\begin{array}{c}-2,22 * \\
(1,33)\end{array}$ & $\begin{array}{c}0,17 \\
(0,40)\end{array}$ \\
\hline Retraso & $\begin{array}{l}-0,10 \\
(0,25)\end{array}$ & $\begin{array}{l}-4,34 \\
(4,11)\end{array}$ & $\begin{array}{l}-0,17 \\
(0,42)\end{array}$ \\
\hline ISEC & $\begin{array}{l}-1,78 * * * \\
(0,57)\end{array}$ & $\begin{array}{c}-0,84 \\
(0,73)\end{array}$ & $\begin{array}{l}-0,39 \\
(0,33)\end{array}$ \\
\hline Subtotal de variables estudiante & $\begin{array}{l}-3,46 \\
(2,78)\end{array}$ & $\begin{array}{c}-24,91^{*} \\
(14,74)\end{array}$ & $\begin{array}{l}-0,21 \\
(0,43)\end{array}$ \\
\hline \multicolumn{4}{|l|}{ Variables centro } \\
\hline Efecto par (ISEC) & $\begin{array}{l}-3,04 * * \\
(1,27)\end{array}$ & $\begin{array}{l}-0,85 \\
(2,69)\end{array}$ & $\begin{array}{l}-0,39 \\
(1,22)\end{array}$ \\
\hline Tamaño colegio & $\begin{array}{c}-0,83 \\
(0,87)\end{array}$ & $\begin{array}{l}-1,76 \\
(6,99)\end{array}$ & $\begin{array}{c}0,32 \\
(1,24)\end{array}$ \\
\hline Alumnos por profesor & $\begin{array}{c}0,57 \\
(0,60)\end{array}$ & $\begin{array}{c}1,92 \\
(10,11)\end{array}$ & $\begin{array}{l}-0,21 \\
(1,10)\end{array}$ \\
\hline Escasez de materiales educativos & $\begin{array}{c}1,33 \\
(1,10)\end{array}$ & $\begin{array}{c}5,88 \\
(10,51)\end{array}$ & $\begin{array}{c}-0,67 \\
(1,26)\end{array}$ \\
\hline Escasez de profesores de matemática & $\begin{array}{c}-0,64 \\
(1,09)\end{array}$ & $\begin{array}{l}-3,50 \\
(7,39)\end{array}$ & $\begin{array}{c}0,77 \\
(1,64)\end{array}$ \\
\hline Porcentaje de profesores titulados & $\begin{array}{c}1,54 \\
(1,05)\end{array}$ & $\begin{array}{l}-8,93 \\
(8,38)\end{array}$ & $\begin{array}{l}-1,19 \\
(1,21)\end{array}$ \\
\hline Montevideo y área metropolitana & $\begin{array}{c}-0,22 \\
(0,30)\end{array}$ & $\begin{array}{c}2,31 \\
(3,08)\end{array}$ & $\begin{array}{c}-0,10 \\
(0,19)\end{array}$ \\
\hline Rural & $\begin{array}{c}0,02 \\
(0,12)\end{array}$ & $\begin{array}{c}0,72 \\
(1,07)\end{array}$ & $\begin{array}{l}-0,03 \\
(0,16)\end{array}$ \\
\hline Subtotal variables centro & $\begin{array}{l}-1,27 \\
(2,49)\end{array}$ & $\begin{array}{c}-4,21 \\
(18,23)\end{array}$ & $\begin{array}{c}-1,50 \\
(2,73)\end{array}$ \\
\hline \multicolumn{4}{|l|}{ Variables institucionales } \\
\hline Selectividad & $\begin{array}{l}-0,15 \\
(0,32)\end{array}$ & $\begin{array}{c}-0,21 \\
(1,24)\end{array}$ & $\begin{array}{c}0,03 \\
(0,21)\end{array}$ \\
\hline Privado & $\begin{array}{c}0,08 \\
(0,17)\end{array}$ & $\begin{array}{l}-2,76 \\
(2,02)\end{array}$ & $\begin{array}{c}-0,15 \\
(0,29)\end{array}$ \\
\hline Subtotal variables institucionales & $\begin{array}{c}-0,07 \\
(0,41)\end{array}$ & $\begin{array}{l}-2,96 \\
(2,14)\end{array}$ & $\begin{array}{c}-0,13 \\
(0,37)\end{array}$ \\
\hline Constante & & $\begin{array}{c}43,31 \\
(26,65)\end{array}$ & \\
\hline \multirow[t]{2}{*}{ Total } & $-4,79$ & $11,23 * * *$ & $-1,84$ \\
\hline & $(4,49)$ & $(3,22)$ & $(2,77)$ \\
\hline
\end{tabular}

Fuente: elaboración propia sobre la base de datos de la Organización de Cooperación y Desarrollo Económicos (OCDE), "PISA 2003" y "PISA 2006" [en línea] http://www.pisa.oecd.org/document/51/0,3746,en_32252351_32235731_39732595_1_1_1_1,00.html

Niveles de significación: *10\%, **5\%,***1\%.

Errores estándar entre paréntesis.

Nota: valores expandidos para toda la población.

ISEC: índice socioeconómico y cultural. 
la pérdida de efectividad en el porcentaje de profesores titulados y la escasez de profesores de matemática. Mientras que la escasez de materiales educativos, la cantidad de alumnos por profesor y las variables ficticias por localidad presentan signo positivo. Con relación a las variables institucionales, la caída en el retorno vinculado a estas se explica básicamente por la evolución del retorno a la condición de colegio privado en 2,8 puntos.

De esta forma, la evolución del efecto retorno de la constante da cuenta de una mayor homogeneidad en los resultados educativos, lo que involucra un importante avance en la equidad de los aprendizajes de los estudiantes uruguayos. Esta situación implica que la mayor parte del incremento en el período es generalizado para todos los estudiantes, especialmente para aquellos que asisten a la Educación Pública. Sin embargo, este efecto equitativo es parcialmente compensado por la caída del retorno a nivel de establecimientos privados, junto con la caída en los retornos a nivel de grados.

Por último, el efecto interacción presenta signo negativo aunque no significativo, y se explica básicamente por la evolución de este efecto en relación con las variables a nivel del centro educativo.

\section{Resultados de la descomposición de Juhn, Murphy y Pierce (1993)}

Para analizar los diferentes efectos de la descomposición de Juhn, Murphy y Pierce, en el cuadro 2 se presentan los valores para cada uno de los efectos considerados, por deciles y en la media. Como se puede observar, el cambio total en los resultados es positivo para los primeros ocho deciles de puntaje, y negativo para los dos últimos, correspondiendo el mayor cambio al segundo, tercero y cuarto decil. El efecto características es negativo para todos los deciles de la distribución de puntajes, aunque de mayor magnitud absoluta para el decil más bajo. Este resultado indica un efecto regresivo relacionado con la magnitud de las características. Según el resultado obtenido al aplicar la metodología de Oaxaca-Blinder, este efecto se concentraría en las variables a nivel individual.

En el cuadro 2 se denota que el cambio total en los resultados es positivo para los primeros ocho deciles de puntaje, y negativo para los dos últimos, siendo el cambio mayor el que corresponde al segundo, tercero y cuarto decil. El efecto características es negativo para todos los deciles de la distribución de puntajes, aunque de mayor magnitud absoluta para el decil más bajo. Este resultado señala un impacto regresivo vinculado a la magnitud de las características. Según el resultado obtenido al aplicar la metodología de Oaxaca-Blinder, este impacto se concentraría en las variables a nivel individual.

En el gráfico 2 es posible observar que el efecto retorno se ubica siempre por sobre el cambio total, y es siempre positivo aunque decreciente. En el cuadro 2 se observa que todos los deciles obtienen incrementos en sus puntajes debido a una mayor eficiencia en el uso de las características, presentando los mayores promedios los dos primeros deciles, lo que indica un impacto progresivo.

CUADRO 2

Descomposición de Juhn, Murphy y Pierce

\begin{tabular}{|c|c|c|c|c|c|}
\hline & Cambio 2006-2003 & Efecto características & Efecto retornos & Efecto residuos & Efecto interacción \\
\hline media & 4,60 & $-6,62$ & 11,23 & 1,92 & $-1,93$ \\
\hline decil 1 & 3,85 & $-13,24$ & 16,48 & 3,49 & $-2,88$ \\
\hline decil 2 & 7,19 & $-8,59$ & 15,04 & 2,98 & $-2,24$ \\
\hline decil 3 & 7,34 & $-6,94$ & 13,40 & 2,72 & $-1,83$ \\
\hline decil 4 & 7,72 & $-5,74$ & 12,54 & 2,95 & $-2,02$ \\
\hline decil 5 & 6,47 & $-6,13$ & 11,05 & 2,63 & $-1,09$ \\
\hline decil 6 & 6,62 & $-4,55$ & 10,72 & 2,20 & $-1,75$ \\
\hline decil 7 & 5,22 & $-4,48$ & 10,18 & 1,78 & $-2,25$ \\
\hline decil 8 & 2,81 & $-5,48$ & 8,56 & 1,33 & $-1,60$ \\
\hline decil 9 & $-0,16$ & $-7,22$ & 7,90 & 0,89 & $-1,72$ \\
\hline decil 10 & $-1,13$ & $-3,77$ & 6,48 & $-1,93$ & $-1,90$ \\
\hline
\end{tabular}

Fuente: elaboración propia sobre la base de datos de la Organización de Cooperación y Desarrollo Económicos (OCDE), “PISA 2003” y “PISA 2006" [en línea] http://www.pisa.oecd.org/document/51/0,3746,en_32252351_32235731_39732595_1_1_1_1,00.html

Nota: valores expandidos para toda la población. 


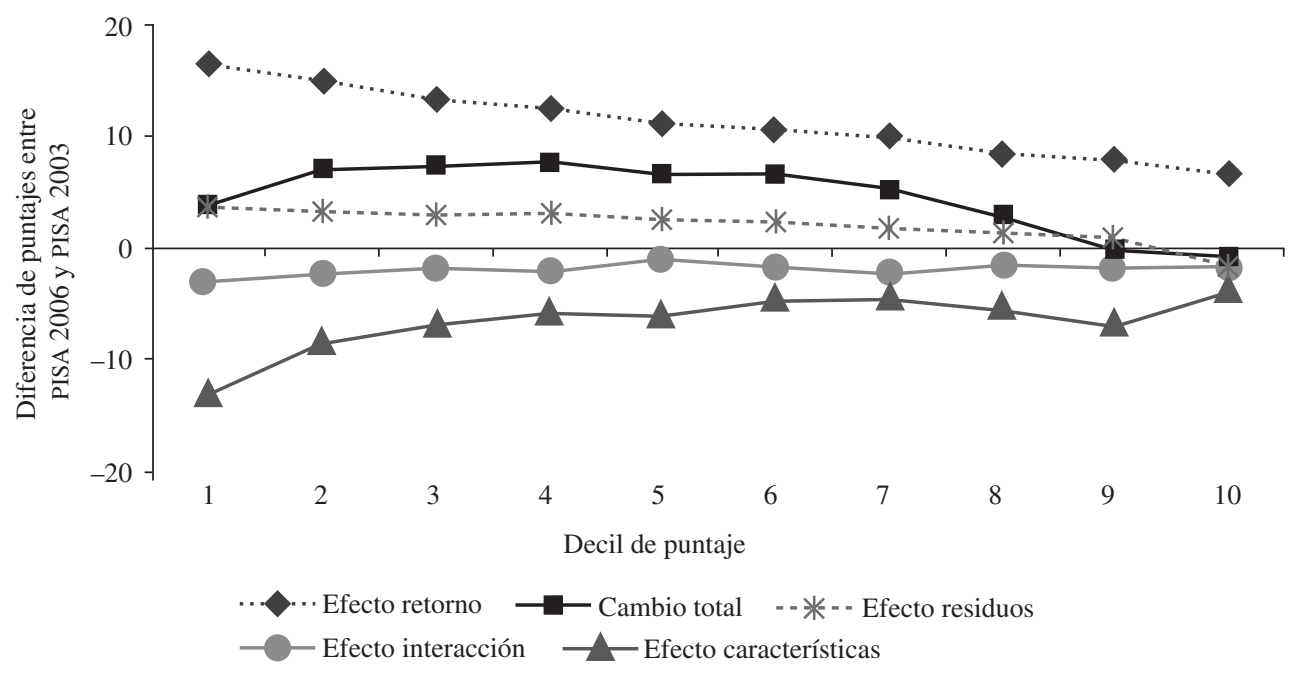

Fuente: elaboración propia sobre la base de datos de la Organización de Cooperación y Desarrollo Económicos (OCDE), PISA 2003 y PISA 2006 [en línea] http://www.pisa.oecd.org/document/51/0,3746,en_32252351_32235731_39732595_1_1_1_1,00.html

El efecto residuos es positivo para los primeros nueve deciles y negativo para el último, aunque de baja magnitud en todos los casos. Este efecto refleja el cambio en la distribución de las variables no observadas, tanto en su magnitud como en sus retornos. A su vez, el efecto interacción es siempre negativo y con mayor magnitud en los deciles intermedios de la distribución.

Los resultados de la descomposición de Juhn, Murphy y Pierce son consistentes con aquellos obtenidos mediante la descomposición de Oaxaca-Blinder. La mayor parte del cambio total entre los puntajes de PISA 2003 y 2006 obedece al efecto positivo del cambio en la eficiencia en el uso de los factores, mientras que el efecto de la magnitud de las características implica un cambio negativo que reduce el impacto positivo de la mayor eficiencia del sistema escolar. En otras palabras, el cambio en el puntaje entre los años 2003 y 2006 se explica por una disminución de los recursos disponibles y un mejoramiento en la eficiencia en el uso de esos recursos.

Los avances en la eficiencia son un reconocimiento de que el país está avanzando en el camino correcto; sin embargo, la reducción de las características plantea un importante desafío al sistema escolar del Uruguay, cual es mejorar las condiciones generales de los estudiantes. Este desafío reviste un carácter de urgencia, puesto que el impacto de la mayor efectividad en el sistema escolar permitiría a los estudiantes más vulnerables avanzar al nivel siguiente de desempeño en matemática en poco más de una década; no obstante, la concentración en la precarización de sus condiciones sociales y económicas durante la misma década implicó que ese plazo se extendiera a tres décadas.

\section{Resultados de la descomposición de Bourguignon, Fournier y Gurgand (1998)}

\section{a) Estimación de las funciones de producción}

Para proceder a efectuar las microsimulaciones, se realiza en primer lugar la estimación de las funciones de producción para las pruebas PISA 2003 y 2006, de forma separada para cada tipo de establecimiento (véase el cuadro 3).

El $R^{2}$ en las estimaciones indica que es posible explicar cerca del $40 \%$ de la varianza del puntaje en el caso de las estimaciones para los centros públicos, mientras que para el caso de los centros privados el $R^{2}$ se ubica en torno del $20 \%$. Esto significa que la capacidad explicativa de las estimaciones para los centros públicos es casi el doble que en el caso de los centros privados, lo que probablemente esté explicado por la composición de estudiantes en cada sector institucional, e implica que el modelo propuesto es más preciso para explicar la evolución del puntaje dentro de los centros públicos.

En primer lugar, los coeficientes asociados a las variables a nivel de estudiante resultan en su mayoría 
CUADRO 3

Estimación de las funciones de producción, centros públicos y privados, 2003-2006

\begin{tabular}{|c|c|c|c|c|}
\hline & \multicolumn{2}{|c|}{ Públicos } & \multicolumn{2}{|c|}{ Privados } \\
\hline & 2003 & 2006 & 2003 & 2006 \\
\hline \multicolumn{5}{|l|}{ Variables estudiante } \\
\hline Sexo $($ mujer $=1)$ & $\begin{array}{c}-18,13^{* * * *} \\
(3,49)\end{array}$ & $\begin{array}{c}-24,59 * * * \\
(3,75)\end{array}$ & $\begin{array}{c}-21,93 * * * \\
(4,95)\end{array}$ & $\begin{array}{c}-21,31 \text { *** } \\
(5,27)\end{array}$ \\
\hline Tercer grado & $\begin{array}{l}41,89 * * * \\
(4,31)\end{array}$ & $\begin{array}{l}34,76^{* * * *} \\
(5,61)\end{array}$ & $\begin{array}{l}110,40 * * * \\
(39,16)\end{array}$ & $\begin{array}{c}-3,49 \\
(26,79)\end{array}$ \\
\hline Cuarto grado & $\begin{array}{c}110,00 * * * \\
(9,45)\end{array}$ & $\begin{array}{l}87,38^{* * * *} \\
(12,96)\end{array}$ & $\begin{array}{l}123,80 * * * \\
(33,57)\end{array}$ & $\begin{array}{c}23,49 \\
(37,79)\end{array}$ \\
\hline Quinto grado & $\begin{array}{l}140,40 * * * \\
(13,04)\end{array}$ & $\begin{array}{l}106,50 * * * \\
(14,68)\end{array}$ & $\begin{array}{l}148,60 \text { *** } \\
(34,99)\end{array}$ & $\begin{array}{c}44,73 \\
(37,31)\end{array}$ \\
\hline Retraso & $\begin{array}{c}-5,53 \\
(8,55)\end{array}$ & $\begin{array}{l}-21,56^{* * *} \\
(10,21)\end{array}$ & $\begin{array}{l}-10,75 \\
(21,50)\end{array}$ & $\begin{array}{l}-26,65 \\
(25,42)\end{array}$ \\
\hline ISEC & $\begin{array}{l}10,88^{* * * *} \\
(1,42)\end{array}$ & $\begin{array}{l}12,80^{* * * *} \\
(1,81)\end{array}$ & $\begin{array}{l}12,78 \text { *** } \\
(4,73)\end{array}$ & $\begin{array}{l}16,15^{* * * *} \\
(3,85)\end{array}$ \\
\hline \multicolumn{5}{|l|}{ Variables centro } \\
\hline Efecto par (ISEC) & $\begin{array}{l}14,50 * * \\
(5,93)\end{array}$ & $\begin{array}{l}15,79 * * * \\
(5,51)\end{array}$ & $\begin{array}{l}36,50 * * \\
(18,21)\end{array}$ & $\begin{array}{l}54,98 \text { *** } \\
(10,96)\end{array}$ \\
\hline Tamaño colegio & $\begin{array}{c}0,00 \\
(0,01)\end{array}$ & $\begin{array}{c}-0,01 \\
(0,01)\end{array}$ & $\begin{array}{c}0,01 \\
(0,02)\end{array}$ & $\begin{array}{c}0,01 \\
(0,02)\end{array}$ \\
\hline Alumnos por profesor & $\begin{array}{l}-0,11 \\
(0,31)\end{array}$ & $\begin{array}{l}-0,14 \\
(0,60)\end{array}$ & $\begin{array}{c}-0,31 \\
(1,26)\end{array}$ & $\begin{array}{c}0,24 \\
(0,91)\end{array}$ \\
\hline Escasez de materiales educativos & $\begin{array}{l}-2,21 \\
(2,50)\end{array}$ & $\begin{array}{l}-1,39 \\
(2,24)\end{array}$ & $\begin{array}{r}-12,07 \\
(8,63)\end{array}$ & $\begin{array}{l}-7,01 \\
(6,31)\end{array}$ \\
\hline Escasez de profesores de matemática & $\begin{array}{c}1,47 \\
(2,17)\end{array}$ & $\begin{array}{l}-0,61 \\
(2,59)\end{array}$ & $\begin{array}{c}4,62 \\
(6,51)\end{array}$ & $\begin{array}{c}1,28 \\
(5,12)\end{array}$ \\
\hline Porcentaje de profesores titulados & $\begin{array}{c}16,05 \\
(13,99)\end{array}$ & $\begin{array}{c}16,84 \\
(11,84)\end{array}$ & $\begin{array}{c}52,14^{*} \\
(27,11)\end{array}$ & $\begin{array}{c}-18,33 \\
(16,04)\end{array}$ \\
\hline Montevideo y área metropolitana & $\begin{array}{l}12,02 * * \\
(4,70)\end{array}$ & $\begin{array}{l}14,14^{* * * *} \\
(4,93)\end{array}$ & $\begin{array}{c}6,87 \\
(13,17)\end{array}$ & $\begin{array}{c}14,55 \\
(14,64)\end{array}$ \\
\hline Rural & $\begin{array}{c}-9,62 \\
(8,43)\end{array}$ & $\begin{array}{c}-5,42 \\
(8,55)\end{array}$ & $\begin{array}{c}20,08 \\
(24,84)\end{array}$ & $\begin{array}{l}0,00 \\
(0)\end{array}$ \\
\hline \multicolumn{5}{|l|}{ Variables institucionales } \\
\hline Selectividad & $\begin{array}{c}12,82 \\
(10,32)\end{array}$ & $\begin{array}{c}6,02 \\
(8,34)\end{array}$ & $\begin{array}{c}1,21 \\
(12,18)\end{array}$ & $\begin{array}{c}3,89 \\
(10,87)\end{array}$ \\
\hline Constante & $\begin{array}{l}346,4 * * * \\
(19,36)\end{array}$ & $\begin{array}{l}390,9 * * * \\
(21,09)\end{array}$ & $\begin{array}{l}325,0 * * * \\
(41,02)\end{array}$ & $\begin{array}{l}432,9 * * * \\
(45,19)\end{array}$ \\
\hline $\begin{array}{l}\text { Observaciones } \\
\mathrm{R}^{2}\end{array}$ & $\begin{array}{r}4679 \\
0,39\end{array}$ & $\begin{array}{r}3826 \\
0,38\end{array}$ & $\begin{array}{r}1156 \\
0,17\end{array}$ & $\begin{array}{r}1013 \\
0,24\end{array}$ \\
\hline
\end{tabular}

Fuente: elaboración propia sobre la base de datos de la Organización de Cooperación y Desarrollo Económicos (ocDE), "PISA 2003" y "PISA 2006" [en línea] http://www.pisa.oecd.org/document/51/0,3746,en_32252351_32235731_39732595_1_1_1_1,00.html

Nota: valores expandidos para toda la población.

Niveles de significación: $* 10 \%, * * 5 \%, * * * 1 \%$.

Errores estándar entre paréntesis.

ISEC: índice socioeconómico y cultural.

significativos. El coeficiente de sexo, negativo y significativo para todos los casos, reduce su magnitud en el período con relación a los centros públicos, y aumenta levemente para los privados. La magnitud de los coeficientes de las variables de grado disminuye en el período para los dos sectores institucionales. Respecto de los centros privados la magnitud y significación de los coeficientes de grado cambia notablemente entre ambos 
años, debido a que parte del coeficiente en el año 2006 está incorporado en la constante, cuya diferencia entre 2003 y 2006 es de más de 100 puntos, lo que indica una mejora para el grupo de control.

Cuando se compara el puntaje adicional de quienes cursan un grado con respecto al anterior, esta diferencia es en todos los casos positiva. A su vez, la ganancia adicional entre grados se aminora para los centros públicos mientras que, en el caso de los privados, la ganancia adicional de quienes cursan cuarto grado respecto del tercero se acrecienta entre 2003 y 2006. El efecto del rezago escolar es negativo en todos los casos, disminuyendo la magnitud del coeficiente en el período para ambos tipos de instituciones. Por otra parte, el coeficiente asociado al ISEC aumenta tanto para los centros públicos como para los privados.

En segundo lugar, las variables a nivel del establecimiento no resultan en su mayoría significativas ${ }^{8}$. Resalta el comportamiento del efecto par, positivo y significativo en todos los casos, que se muestra creciente durante el período. Este coeficiente se acrecienta en casi 50\% en los colegios privados, mientras que en los públicos el aumento se sitúa en torno de un 9\%. Asimismo, el coeficiente asociado al ISEC a nivel individual y el efecto par muestran un comportamiento altamente diferenciado entre colegios públicos y privados. Mientras que en el primer caso el efecto par es 1,3 veces superior al coeficiente del ISEC a nivel individual, en los colegios privados resulta casi 3 veces superior. Estas diferencias entre el sector público y privado se traducen en mayores incentivos para una mayor segregación en los centros privados, de modo de mantener un determinado nivel sociocultural del conjunto de los alumnos que asisten al centro y recibir, por lo tanto, el premio por segregación.

En lo que respecta al tamaño de la localidad, la variable ficticia asociada a Montevideo presenta signo positivo, aumentando en el período, mientras que la variable ficticia asociada a localidades rurales es negativa para los centros públicos y positiva para los privados. La variable institucional de selectividad se reduce entre

\footnotetext{
${ }^{8}$ Dado que en las estimaciones se utilizaron algunos controles que podrían, eventualmente, tener un efecto no lineal, cabría la posibilidad de que la no significancia estadística observada en algunos de ellos pudiera relacionarse con la especificación lineal. En este sentido, se testeó la no linealidad en el tamaño del establecimiento y del tamaño del curso de los estudiantes, pero los resultados no se modificaron mayoritariamente, con la excepción del año 2006 con respecto al tamaño de los colegios, en que aparece un efecto decreciente a partir de los colegios con 800 o más estudiantes, que son una minoría en el total muestral.
}

2003 y 2006 para los centros públicos, y tiene el comportamiento inverso en el caso de los privados.

Finalmente, el valor de la constante es siempre positivo y significativo, y su coeficiente aumenta entre ambos años.

b) Estimación de la elección del tipo de centro educativo

A continuación se estima una función logit para cada año, donde el valor 1 corresponde al caso en que el estudiante asiste a Educación Pública. Los resultados se presentan en el cuadro 4.

La variable ficticia de sexo resulta negativa, pero no significativa, para explicar la probabilidad de concurrir a un centro público en relación con la probabilidad de concurrir a uno privado. En cambio, el efecto del rezago escolar resulta siempre positivo y significativo con respecto a la probabilidad de asistir a un centro público, y negativo en el caso de la probabilidad de asistir a uno privado, mientras que el ISEC resulta negativo si se da la probabilidad de asistir a un centro público.

CUADRO 4

Estimación logit para la elección del tipo de centro educativo, 2003-2006

\begin{tabular}{lcc}
\hline & 2003 & 2006 \\
\hline Sexo (mujer = 1) & $-0,19$ & $-0,09$ \\
Retraso & $(0,15)$ & $(0,10)$ \\
& $1,53^{* * *}$ & $1,10^{* * *}$ \\
ISEC & $(0,36)$ & $(0,29)$ \\
& $-1,36^{* * *}$ & $-1,44 * * *$ \\
Tamaño del colegio & $(0,11)$ & $(0,10)$ \\
& 0,00 & 0,00 \\
Alumnos por profesor & $(0,00)$ & $(0,00)$ \\
& 0,07 & $0,08^{* *}$ \\
Montevideo y área metropolitana & $(0,05)$ & $(0,04)$ \\
& $-1,93^{* * *}$ & $-1,71^{* * *}$ \\
Rural & $(0,37)$ & $(0,41)$ \\
& 0,41 & \\
Constante & $(1,42)$ & $1,29 * *$ \\
& $1,35 * *$ & $(0,55)$ \\
\hline Observaciones & $(0,68)$ & 50,18 \\
Estadístico F & 835 & 0,00 \\
Prob $>\mathrm{F}$ & 32,95 & 4381 \\
\hline
\end{tabular}

Fuente: elaboración propia sobre la base de datos de la Organización de Cooperación y Desarrollo Económicos (OCDE), "PISA 2003" y "PISA 2006" [en línea] http://www.pisa.oecd.org/document/51/0,374 6,en_32252351_32235731_39732595_1_1_1_1,00.html

Nota: valores expandidos para toda la población.

Niveles de significación: $* 10 \%, * * 5 \%, * * * 1 \%$.

Errores estándar entre paréntesis.

Valor 1= Educación Pública.

ISEC: índice socioeconómico y cultural.

Prob > F: $\mathrm{p}$ valor asociado al estadístico $\mathrm{F}$, usado para testear la hipótesis nula de que todos los coeficientes del modelo son 0 . 
Por su parte, el tamaño del colegio y la cantidad de alumnos por profesor muestran un efecto casi nulo en la probabilidad de asistencia a un centro público. Las variables vinculadas al tamaño de la localidad indican que vivir en Montevideo tiene un efecto negativo y significativo en la probabilidad de asistencia a un centro público, mientras que vivir en una zona rural no resulta significativo en el año $2003^{9}$.

En conclusión, los estudiantes que se concentran en las escuelas públicas tienden a provenir de familias más vulnerables, presentar un menor éxito académico y vivir en ciudades más pequeñas.

\section{Resultado de las microsimulaciones}

Los principales resultados de las microsimulaciones se resumen en el cuadro 5. La información presentada contiene los efectos en los cambios tanto en el promedio como en los deciles de puntaje, como resultado de la diferencia entre la distribución simulada para cada caso y la observada en el año 2003. Asimismo, en el cuadro A.3 del anexo se presentan los efectos cuando se consideran cambios únicamente en un tipo de colegio.

${ }^{9}$ No se dispone del coeficiente correspondiente al año 2006 debido a que la muestra de ese año no presenta observaciones de centros privados en zonas rurales.

\section{a) Efecto características}

El efecto características en su conjunto es el efecto positivo de mayor importancia para explicar el cambio total en el puntaje de la prueba PISA Matemática entre los años 2003 y 2006. En el promedio, si en 2003 los centros educativos e institucionales tuvieran la misma dotación de recursos individuales que en 2006, el puntaje se incrementaría en 9,2 puntos (véase el cuadro 5).

Sin embargo, cabe hacer una advertencia sobre la correcta interpretación de este efecto. La simulación de características implica imponer en el año 2006 el porcentaje no ponderado de los casos que cumplen con una determinada característica para las variables dicotómicas o categóricas, o la media no ponderada para las variables continuas. Por otra parte, cuando se calcula el efecto características las medidas de distribución son calculadas utilizando el ponderador muestral del año 2003, que — como será comentado más adelante- presenta gran variabilidad respecto de aquel del año 2006, debido a cambios importantes en el diseño de la muestra de ambos años. En este sentido, una correcta interpretación de la evolución de los recursos en el período debería incluir no solo la simulación de las características - pesadas con el factor de expansión del año 2003-, sino también el peso muestral del año que se intenta simular. Es posible entonces analizar lo que sucede cuando solo se simulan las características del año 2006 y luego comparar este resultado con el obtenido cuando se simulan además los pesos muestrales de ese

CUADRO 5

Resultados de las microsimulaciones promedio y por decil de puntaje, 2003-2006

\begin{tabular}{|c|c|c|c|c|c|c|c|c|c|c|c|}
\hline & Promedio & 1 & 2 & 3 & 4 & 5 & 6 & 7 & 8 & 9 & 10 \\
\hline PISA Matemática 2003 & 422,20 & 257,03 & 318,07 & 355,44 & 384,22 & 410,99 & 435,48 & 460,99 & 489,39 & 523,25 & 587,56 \\
\hline PISA Matemática 2006 & 426,80 & 260,88 & 325,26 & 362,79 & 391,94 & 417,46 & 442,10 & 466,22 & 492,20 & 523,09 & 586,43 \\
\hline Diferencia total PISA Matemática (2006-2003) & 4,60 & 3,85 & 7,19 & 7,34 & 7,72 & 6,47 & 6,62 & 5,22 & 2,81 & $-0,16$ & $-1,13$ \\
\hline Efecto características & 9,24 & 13,07 & 17,87 & 15,48 & 13,40 & 10,77 & 8,50 & 5,23 & 2,98 & 2,43 & 2,85 \\
\hline Efecto peso & $-2,76$ & $-4,31$ & $-5,02$ & $-3,91$ & $-3,02$ & $-3,06$ & $-2,59$ & $-2,04$ & $-1,86$ & $-1,06$ & $-0,67$ \\
\hline Efecto características+peso & $-2,11$ & 0,50 & 0,77 & $-0,11$ & $-1,53$ & $-2,66$ & $-2,76$ & $-4,37$ & $-6,07$ & $-4,31$ & $-0,64$ \\
\hline Efecto precio & 7,69 & 13,24 & 12,15 & 10,37 & 9,21 & 7,44 & 6,91 & 6,12 & 4,67 & 3,90 & 2,67 \\
\hline Efecto características+peso+precio & 7,59 & 17,40 & 16,54 & 12,63 & 10,22 & 7,51 & 4,42 & 2,93 & 0,35 & 0,97 & 2,86 \\
\hline Efecto choice & $-0,05$ & $-0,03$ & 0,02 & 0,04 & $-0,03$ & $-0,01$ & $-0,03$ & $-0,01$ & $-0,02$ & $-0,02$ & $-0,29$ \\
\hline Efecto características+peso+precio+choice & 5,91 & 9,90 & 10,11 & 7,61 & 7,22 & 6,22 & 5,60 & 4,08 & 2,74 & 2,39 & 3,20 \\
\hline Efecto residuos & 0,00 & 2,58 & 1,62 & 1,09 & 1,07 & 0,68 & 0,28 & 0,05 & $-0,92$ & $-2,03$ & $-4,38$ \\
\hline Efecto características+peso+precio+choice+residuos & 5,93 & 13,53 & 11,63 & 8,44 & 7,89 & 6,50 & 5,90 & 4,21 & 2,00 & 0,65 & $-1,18$ \\
\hline
\end{tabular}

Fuente: elaboración propia sobre la base de datos de la Organización de Cooperación y Desarrollo Económicos (OCDE), "PISA 2003” y “PISA 2006" [en línea] http://www.pisa.oecd.org/document/51/0,3746,en_32252351_32235731_39732595_1_1_1_1,00.html

Nota: valores expandidos para toda la población.

Efecto choice: efecto selección de establecimiento. 
año y, por lo tanto, se refleja más fielmente la población que se intenta simular.

El efecto de la simulación solo de las características es mayor para los primeros deciles de la distribución y disminuye para los deciles superiores, lo que implica un efecto redistributivo al beneficiar a los alumnos de más bajo desempeño educativo. Si se analiza este efecto distinguiendo según el tipo de institución en la que se simulan las características del año 2006 (véase el cuadro A.3 del anexo), se observa que el cambio más acentuado se produce para los centros públicos ( 8,4 puntos), siendo estos los que explican la casi totalidad del efecto características, ya que el aporte que realizan los centros privados es de tan solo 0,8 puntos.

En el cuadro A.4 del anexo se presenta el detalle del efecto características relacionado con cada variable incluida en el modelo y con los grupos de variables definidos, distinguiendo entre tipo de centro educativo. Como se puede observar, el incremento de los recursos educativos se concentra en las variables individuales (7 puntos) y se vincula principalmente a la cantidad de estudiantes que cursan cuarto grado y a los colegios públicos, siendo mayor para los deciles superiores de la distribución. La variable de retraso del estudiante también presenta un efecto positivo aunque reducido. Por su parte, la variable ficticia de sexo, así como los demás grados de escolaridad y el ISEC, indican un efecto negativo en la evolución del resultado de la prueba PISA de Matemática.

A su vez, las variables del centro educativo explican un cambio positivo de 2,3 puntos, destacándose el porcentaje de profesores titulados (1,6 puntos). El efecto par asociado indica un cambio positivo de 0,2 puntos, mientras que el ISEC a nivel individual tiene una repercusión negativa y casi nula. La cantidad de alumnos por profesor, la escasez de materiales educativos y la variable ficticia asociada a Montevideo también presentan un efecto positivo, mientras que el tamaño del colegio, la escasez de profesores de matemática y la variable ficticia asociada a zona rural presentan un efecto negativo. Lo mismo sucede con la variable institucional de selectividad, que registra un efecto negativo (véase el cuadro A.4).

\section{b) Efecto peso}

Cuando se ajustan los pesos individuales en la muestra correspondiente a 2003, a objeto de reflejar la población del año 2006, el cambio total del resultado de la prueba PISA disminuye en 2,8 puntos (véase el cuadro 5). Este efecto se explica fundamentalmente por el cambio en el diseño muestral de ambas pruebas. En el caso del Uruguay hubo varios cambios en el diseño de estratos entre las pruebas PISA de 2003 y 2006. En el análisis de la primera de estas pruebas se consideraron 8 estratos en la definición del diseño de la muestra, mientras que en el siguiente año se consideraron 16 estratos. Asimismo, hubo cambios en la tasa de respuesta en los centros y en la cantidad y tipo de centros que no aplicaron o no lo hicieron de la forma correcta (ANEP, 2007b). Por otra parte, como fue comentado anteriormente, entre las pruebas consideradas de ambos años hubo cambios en la tasa de asistencia, especialmente en las localidades más pequeñas. Todos estos factores generaron variaciones en los pesos muestrales que explican la magnitud del efecto peso obtenido mediante la microsimulación.

El efecto negativo del peso resulta de mayor importancia en los primeros deciles de la distribución, y se explica fundamentalmente por el cambio en el peso de los colegios públicos (véase el cuadro A.3 del anexo).

\section{c) Interacción del efecto características y peso}

Cuando se combina el cambio de las características con el cambio en el peso (tercera simulación en el cuadro 5), el efecto promedio disminuye de magnitud, pero mantiene el signo negativo del efecto peso ( $-2,1$ puntos). Como ya se mencionó, el cambio en este resultado obedece a la variabilidad del peso muestral en ambos años y a las modificaciones del diseño de la muestra, y estaría reflejando la verdadera evolución de los recursos en el período.

$\mathrm{Al}$ analizar este efecto por deciles se observa que los primeros dos deciles de la distribución presentan signo positivo, mientras que los otros ocho mantienen el signo negativo, por lo que el efecto conjunto tiene un gran carácter redistributivo. Así como sucede con el efecto peso aisladamente, la combinación de estos dos efectos es también negativa en el caso de los colegios públicos, mientras que en los privados este signo es positivo.

\section{d) Efecto retorno}

El efecto retorno proviene de la simulación de los coeficientes del año 2006 en la distribución de resultados del año 2003. Como se observa en el cuadro A.5, el efecto total de este componente alcanza a un incremento de 7,7 puntos y resulta positivo para todos los deciles de la distribución, con mayor magnitud para los primeros, lo que nuevamente indica un efecto redistributivo. El signo de este efecto se explica por el aumento en la eficiencia de las características para los colegios públicos, presentando un efecto negativo en el caso de los privados (véase el cuadro A.3).

Al analizar separadamente el efecto retorno de cada una de las variables de la función de producción 
(véase el cuadro A.5), se observa que el principal factor es la mayor efectividad de la constante, que alcanza a 53,4 puntos, lo que indica un mejoramiento generalizado de la eficiencia de los estudiantes.

El efecto del conjunto de variables a nivel individual, del centro e institucionales presenta signo negativo, resultado consistente con lo obtenido mediante la descomposición de Oaxaca-Blinder. Destaca el aumento en la eficiencia del efecto par (1,3 puntos), mientras que el ISEC a nivel individual ve disminuida su efectividad. A su vez, la mayor parte del incremento del efecto par corresponde a los centros privados, mientras que los públicos vieron disminuir su efectividad en este factor.

Otras variables a nivel del centro educativo que tienen una repercusión positiva en el efecto retorno son la cantidad de alumnos por profesor, la escasez de materiales educativos y las variables de región. Mientras que el tamaño del colegio, la escasez de profesores de matemática y el porcentaje de profesores titulados presentan un efecto negativo. Por su parte, la variable institucional de selectividad académica también presenta un efecto negativo.

A nivel del estudiante, las variables generan un efecto total negativo de 36,5 puntos, y es el sector público el que explica la mayor parte de este cambio ( $-22,6$ puntos). Dentro de las variables a nivel individual, el cambio más sustantivo proviene de la variable ficticia de cuarto grado $(-22,5)$, con una mayor repercusión negativa en los deciles más altos de la distribución. El resto de las variables a nivel individual presentan todas un efecto retorno negativo, aunque de menor magnitud. Por último, la variable institucional de selectividad registra también un efecto negativo.

e) Interacción de los efectos características y retorno En el gráfico 3 se presenta el efecto combinado del cambio en las características y coeficientes para todos los deciles de la distribución. Según se observa, este efecto es positivo para toda la distribución y mayor para los primeros deciles.

\section{f) Interacción de los efectos características, retorno y peso}

En la quinta simulación del cuadro 5 se presenta el efecto combinado de la simulación de características, coeficientes y peso. En este caso el efecto promedio disminuye respecto de la simulación aislada de características o coeficientes (7,6 puntos). Asimismo, el efecto combinado resulta de mayor magnitud para los primeros deciles de la distribución, lo que indica nuevamente un efecto redistributivo. Al analizar el cambio por tipo de centro educativo (véase el cuadro A.3 del anexo) se aprecia que los colegios públicos presentan la mayor magnitud en este efecto.

GRÁFICO 3

Efectos combinados por decil de puntaje

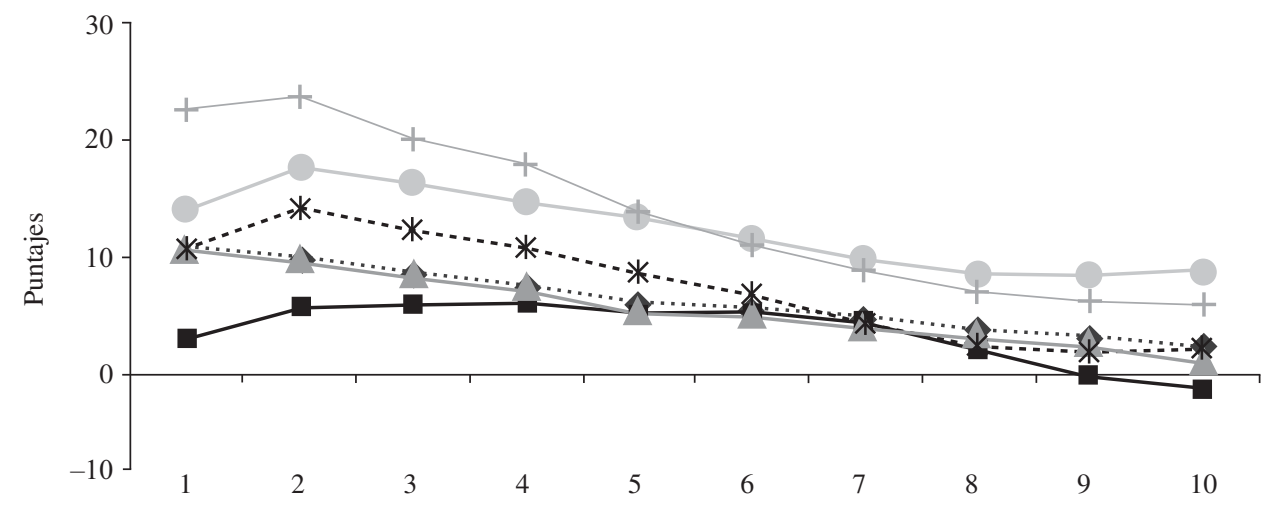

Decil de puntaje

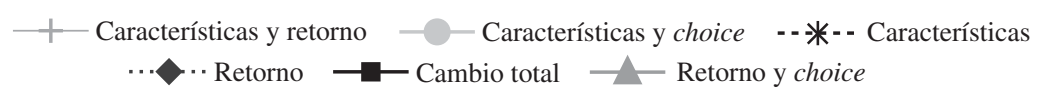

Fuente: elaboración propia sobre la base de datos de la Organización de Cooperación y Desarrollo Económicos (ocDE), PISA 2003 y PISA 2006 [en línea] http://www.pisa.oecd.org/document/51/0,3746,en_32252351_32235731_39732595_1_1_1_1,00.html

Efecto choice: efecto selección de establecimiento. 


\section{g) Efecto elección de establecimiento}

El efecto elección de establecimiento o efecto choice resulta de signo negativo en el promedio y muy cercano a cero (0), como se observa en el cuadro 5. El efecto es prácticamente igual para todos los deciles de la distribución.

h) Interacción de los efectos características, retorno, elección de establecimiento y peso

Cuando se realiza la simulación combinada de las características, coeficientes, elección de establecimiento y peso, el efecto promedio se reduce a 5,9 puntos (véase el cuadro 5), presentando las mayores disminuciones con respecto a la simulación combinada anterior en los primeros deciles de la distribución (véase el gráfico 4). En este caso, mientras que los colegios públicos presentan signo positivo en este efecto combinado, los colegios privados tienen el signo contrario.

\section{i) Efecto residuos}

El efecto de simular los residuos del año 2006 en el año 2003 da como resultado el promedio nulo (véase el cuadro 5), comportamiento que se produce para todos los tipos de instituciones (véase el cuadro A.3 del anexo). Sin embargo, el efecto residuos presenta un comportamiento diferenciado según los deciles de la distribución, teniendo signo positivo en los primeros siete deciles y negativo en los últimos tres. j) Efectos conjuntos de las microsimulaciones

El efecto conjunto de las microsimulaciones (última simulación del cuadro 5) indica un pequeño cambio en el promedio, con una disminución de la desigualdad. El carácter progresivo del cambio total se explica por el resultado de los centros públicos ${ }^{10}$. A su vez, los resultados señalan que el puntaje solo mejora para estos últimos, ya que la evolución es negativa para los privados (véase el cuadro A.3 del anexo). El principal avance se refiere a un mejoramiento generalizado de la eficiencia en el uso de los recursos. Por otra parte, este mejor resultado es compensado por un efecto negativo en cuanto a dotación de recursos.

Cuando se incluye la simulación de las variables no observables en las cuatro primeras simulaciones es posible explicar una parte importante de la desigualdad en la evolución de los promedios de puntaje. El efecto conjunto es superior para los primeros deciles y se vuelve negativo para los dos últimos. Este resultado es consistente con el impacto progresivo del efecto residuos obtenido al aplicar la metodología de Juhn, Murphy y Pierce.

\footnotetext{
${ }^{10} \mathrm{Si}$ se calcula el efecto conjunto de las microsimulaciones por decil de puntaje, considerando el cambio en un solo tipo de colegio, se encuentra un efecto progresivo para los centros públicos y un efecto regresivo para los privados, lo que es consistente con lo observado en el cambio total de puntaje por tipo de institución.
}

GRÁFICO 4 Todos los efectos por decil de puntaje

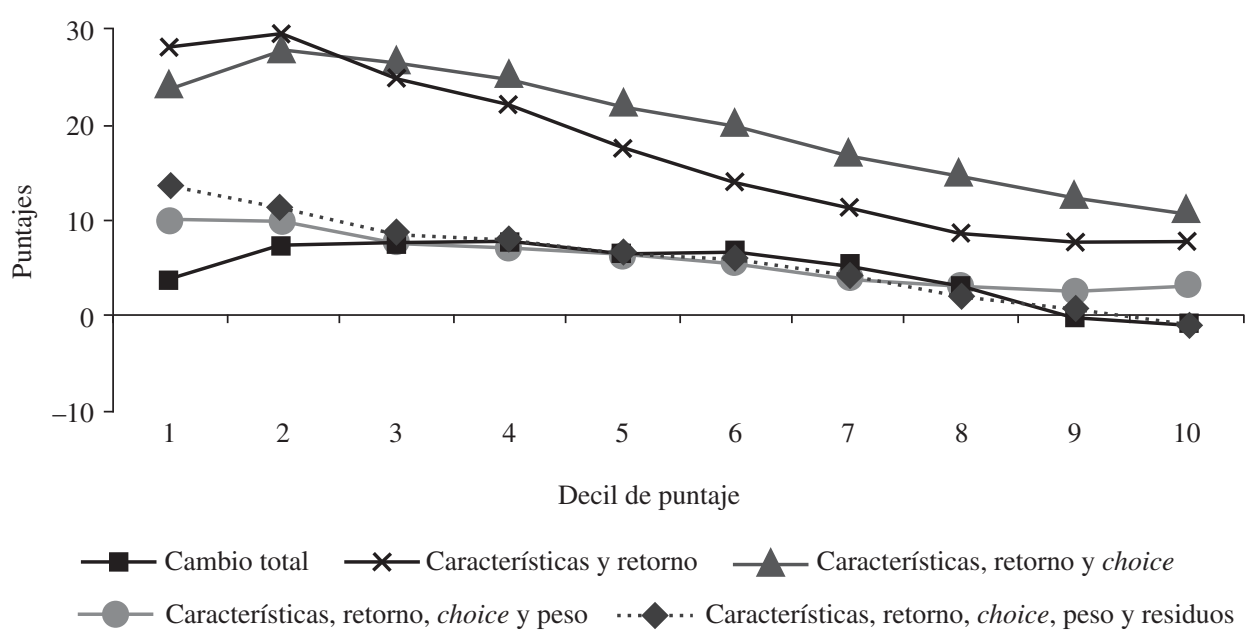

Fuente: elaboración propia sobre la base de datos de la Organización de Cooperación y Desarrollo Económicos (OCDE), "PISA 2003" y "PISA 2006" [en línea] http://www.pisa.oecd.org/document/51/0,3746,en_32252351_32235731_39732595_1_1_1_1,00.html

Efecto choice: efecto selección de establecimiento. 
Para analizar la evolución de la magnitud de la reducción en la desigualdad de los resultados se estiman el coeficiente de Gini y los índices de Theil y de entropía, utilizados en forma generalizada para analizar la evolución en la distribución del ingreso. Como se advierte en el cuadro A.6 del anexo, la evolución de los tres indicadores da cuenta de una disminución de los niveles de desigualdad, aunque reducida, en los puntajes de matemática. La magnitud de esta reducción alcanza entre $0,1 \%$ y $0,4 \%$ de estos indicadores, lo que se explica sobre todo por el efecto progresivo de la simulación de características y precios, y particularmente por el cambio en los colegios públicos. El efecto de los residuos también repercute progresivamente en la desigualdad, aunque con menor magnitud. El efecto conjunto de todas las simulaciones denota una disminución de $0,8 \%$ en el índice de Gini, y de 0,3\% en los índices de Theil y de entropía.

Al combinar todas las simulaciones, el efecto promedio alcanza a 5,9 puntos (de 4,6 que es el cambio observado). El ajuste total es más exacto para últimos deciles de la distribución, mientras que se vuelve más inexacto en el primer decil (véase el gráfico 4). El diseño muestral del año 2006 resulta más complejo y exacto a la hora de reflejar la población estudiantil en el tramo de edad considerado, por lo que al simular el peso de dicho año en la muestra de 2003 —conjuntamente con las características, retornos, elección de establecimiento y residuos - es posible explicar los cambios en el $90 \%$ de la distribución, permitiendo identificar con claridad los principales factores vinculados a la evolución de cada uno de los deciles de puntaje.

\section{V}

\section{Comparación de los resultados}

La aplicación de las tres estrategias metodológicas arroja resultados consistentes entre sí, permitiendo una complementación en el grado de complejidad del análisis y aportando cada una de ella un valor adicional.

Los resultados obtenidos al aplicar la metodología de Oaxaca-Blinder indican que el cambio total en el puntaje se explica fundamentalmente por una mayor eficiencia generalizada del sistema escolar en el uso de los recursos disponibles. El aumento en el retorno de la constante es el principal factor explicativo del incremento de la eficiencia. Este aumento generalizado para todos los estudiantes - especialmente en los centros públicos - apunta a una mayor homogeneidad en los resultados educativos y se debe sobre todo a la recuperación económica en los años considerados. Sin embargo, este efecto positivo es atenuado por una menor eficiencia en las variables más importantes a nivel del estudiante y centro, principalmente los retornos a los diferentes grados de escolaridad.

Por su parte, en el año 2006 las características resultan más desventajosas que en el año 2003, hecho que se concentra principalmente en las variables relativas al estudiante. Si bien este hecho contrasta con un período de recuperación económica, puede deberse a diversos factores como los cambios en la matrícula o los cambios demográficos que experimentó el país en estos años.

La metodología de Juhn, Murphy y Pierce permite además obtener conclusiones sobre la repercusión de los efectos en los distintos deciles de la distribución, indicando un efecto progresivo como consecuencia del efecto retorno.

Por otra parte, las microsimulaciones permiten identificar la relevancia de las diferencias entre estudiantes que asisten a colegios públicos y privados, puesto que el cambio observado en el período se concentra solo en los estudiantes que asistieron a entidades estatales. Asimismo, las estimaciones realizadas dan cuenta de la sensibilidad de los resultados frente a cambios en la composición muestral. El efecto características resulta de signo positivo cuando se realizan las microsimulaciones, a diferencia de lo obtenido con las otras dos técnicas de descomposición. Sin embargo, cuando se incorpora la simulación del peso muestral, el efecto conjunto resulta negativo y de magnitud muy similar al obtenido previamente con Oaxaca-Blinder y Juhn, Murphy y Pierce. 


\section{VI}

\section{Conclusiones}

En este estudio se brinda información sobre la existencia de diferencias en la distribución de resultados en las pruebas PISA de Matemática 2003 y 2006, identificando factores que determinan los cambios en los resultados y la evolución de la incidencia de estos factores en el período. El recorrido por las tres metodologías aplicadas permite entregar conclusiones consistentes entre ellas, siendo posible afirmar que el país presenta un piso insatisfactorio en cuanto a equidad y resultados.

En primer lugar se puede concluir que, si bien el cambio observado entre los años 2003 y 2006 es muy pequeño, sin mayor repercusión en el mejoramiento del desempeño en matemática entre los estudiantes uruguayos, existen cambios internos en las características y retornos que se contraponen y generan un escaso cambio total en el período, lo que verifica la hipótesis inicial del trabajo.

En segundo lugar, existe evidencia de que la mejora en el puntaje entre ambos años se debe a un aumento en la capacidad de la educación de transformar en logros de aprendizaje los recursos y características educativas, y en particular, a un mejoramiento generalizado en la eficiencia en el uso de los recursos derivados de la recuperación económica en el período de estudio, que beneficia especialmente a los centros públicos, aunque compensada parcialmente por una disminución en la eficiencia de efectos individuales de grado.

En tercer lugar, se produce una disminución en la dotación de recursos, que se concentra especialmente en las variables a nivel del estudiante, en particular en las características socioeconómicas y culturales y el porcentaje de estudiantes que cursan los grados más avanzados, situación que es aún más crítica entre los estudiantes más vulnerables. De esta forma, la reducción en las condiciones familiares y escolares entre los estudiantes más vulnerables implicó que disminuyera la velocidad con que estos jóvenes pudieran pasar al nivel siguiente de la escala construida por la prueba PISA para medir las competencias en matemática. De no haberse presentado esta situación, este grupo hubiese logrado el objetivo en poco más de una década, mientras que al incorporar ambos grupos de factores tal objetivo requerirá de 25 a 30 años.

Por último, es posible concluir que los mejores resultados de la prueba PISA de Matemática tuvieron un efecto redistributivo y beneficiaron sobre todo a aquellos estudiantes con inferiores desempeños educativos. Este resultado refleja una aminoración en la dispersión de los puntajes, y se explica por la evolución dentro de los colegios públicos.

El crecimiento económico debiese estar en estrecha relación con un sustantivo incremento en el desempeño, lo que no se percibe en los resultados obtenidos por el Uruguay en el período, lo que refleja que el deterioro social y económico de comienzos de la década redujo en forma estructural las posibilidades educativas de los jóvenes estudiantes, mientras que los beneficios de la recuperación probablemente solo serán observados a partir de los resultados de la prueba PISA de 2012. Las metodologías aplicadas permiten indagar en profundidad dónde están los desafíos y condiciones de los estudiantes uruguayos, y la forma de resolver la deuda del país en cuanto a brindar una educación satisfactoria para todos. Es posible concluir que los esfuerzos deben enfocarse en sumar recursos educativos y aumentar la eficiencia a nivel individual. Resultan primordiales las políticas orientadas a ofrecer mayor apoyo a los alumnos de contextos socioculturales desfavorecidos, a objeto de reducir la elevada tasa de repitencia escolar y propiciar la búsqueda de mejores retornos por año adicional de educación.

A pesar de la caída del ISEC, el puntaje promedio mejora en el período y, si bien este aumento es marginal, hay un crecimiento sustantivo en los deciles medio-bajos y medios. Este resultado indicaría que luego del impacto (shock) económico negativo experimentado por el país, que afecta con mayor intensidad a los grupos vulnerables de la sociedad, no solo en términos económicos, sino también en oportunidades sociales y de movilidad como son los aprendizajes, en el período 2003-2006 parece haberse revertido la potencial pérdida provocada por la crisis. Sin embargo, en este escenario se plantean importantes desafíos para mejorar la efectividad del sistema escolar en su conjunto, puesto que el avance registrado en el período 2003-2006 podría evidenciar tan solo una recuperación y no la existencia de un sistema escolar más efectivo, lo que hace suponer que no se pueden esperar nuevos incrementos en los resultados futuros de la prueba PISA, tal como ha sucedido con los resultados del año 2009.

La presente investigación abre la posibilidad de aplicar estas metodologías a los datos de la prueba PISA 
de 2009 para entender con más detalle la historia detrás de los resultados obtenidos por el Uruguay. Los datos más recientes permitirán ampliar el análisis con la evolución de los últimos años, frente a un crecimiento económico y reformas educativas enfocadas a mejorar la educación y volverla más equitativa. Asimismo, con miras a futuras ampliaciones de la investigación, resulta interesante comparar el resultado obtenido por el Uruguay con los de otros países de niveles socioeconómicos similares como Chile o la Argentina, y respecto de países que han logrado avanzar a situaciones mucho mejores, como es el caso de Polonia.

ANEXO

CUADRO A.1

Variables seleccionadas

\begin{tabular}{ll}
\hline Variable utilizada & Comentario \\
\hline Variable de resultados & \\
\hline Puntaje en Matemática & Puntaje PISA 2003-2006, 5 valores plausibles para el resultado en Matemática \\
\hline Variables del estudiante & \\
\hline $\begin{array}{l}\text { Sexo } \\
\text { Grado }\end{array}$ & $\begin{array}{l}\text { Variable ficticia para el sexo del alumno (categoría omitida: hombre) } \\
\text { Cinco variables ficticias para el grado que se encuentra cursando el alumno: grado } 7 \text { (primer } \\
\text { año), grado } 8 \text { (segundo año), grado } 9 \text { (tercer año), grado } 10 \text { (cuarto año), grado } 11 \text { (quinto } \\
\text { año) (categorías omitidas: no pertenece a ese grado) } \\
\text { Variable ficticia que indica si el estudiante ha repetido algún curso (categoría omitida: } \\
\text { sin retraso) } \\
\text { Variable elaborada por ocDE/PISA (ESCS - Index of Economic, Social and Cultural Status) } \\
\text { en que se considera la educación y ocupación de los padres, y los bienes en el hogar. Nor- } \\
\text { malizada con media 0 y desvío estándar } 1 \text { para el promedio de los países de la ocDE. Un } \\
\text { valor del índice mayor es sinónimo de un contexto socioeconómico más elevado }\end{array}$ \\
\hline
\end{tabular}

Variables del establecimiento

Efecto par

Tamaño del colegio

Alumnos por profesor

Escasez de materiales educativos

Escasez de profesores de matemática calificados

Porcentaje de profesores titulados

Tamaño de la localidad

Variables institucionales

Selectividad académica

Secundaria pública

Secundaria privada
Variable que mide el promedio del ISEC de los alumnos del mismo centro

Variable continua que indica la cantidad promedio de alumnos matriculados

Variable continua que indica la cantidad de alumnos promedio por profesor

Variable que indica el grado en que la capacidad del centro educativo para brindar enseñanza es afectada por la disponibilidad de material de enseñanza escasa o inadecuada: escala con rango 1 a 4

Variable que indica el grado en que la capacidad del centro educativo para brindar enseñanza es afectada por la no disponibilidad de profesores de matemática calificados: escala con rango 1 a 4

Variable que indica la proporción de docentes titulados en el centro educativo: escala con rango 0 a 1

Cuatro variables ficticias que indican la ubicación del establecimiento: Montevideo y área metropolitana, ciudades capitales del interior del país, ciudades menores del interior del país, rural (categorías omitidas: no pertenece a esa localidad)

Variable ficticia que indica si el establecimiento aplica criterios académicos de selección, construida a partir del índice de selectividad del centro educativo elaborado por OCDE/PISA (categoría omitida: no aplica)

Variable ficticia que indica si la institución corresponde a secundaria pública — general, militar, rural y técnica- (categoría omitida: no corresponde)

Variable ficticia que indica si la institución corresponde a secundaria privada (categoría omitida: no corresponde)

Fuente: elaboración propia. 
Estadísticas descriptivas, 2003-2006

\begin{tabular}{|c|c|c|c|c|c|c|c|c|c|c|}
\hline \multirow[b]{3}{*}{ Puntaje en Matemática } & \multicolumn{2}{|c|}{ Media } & \multicolumn{2}{|c|}{$\begin{array}{l}\text { Desviación } \\
\text { estándar }\end{array}$} & \multicolumn{4}{|c|}{ Rango } & \multicolumn{2}{|c|}{ Puntaje } \\
\hline & 2003 & 2006 & 2003 & 2006 & \multicolumn{2}{|c|}{2003} & \multicolumn{2}{|c|}{2006} & 2003 & 2006 \\
\hline & 422,20 & 426,80 & 95,22 & 93,37 & 108,93 & 734,41 & 102,58 & 732,04 & & \\
\hline \multicolumn{11}{|l|}{ Variables estudiante } \\
\hline Sexo $($ mujer $=1)$ & 0,51 & 0,51 & 0,50 & 0,50 & 0,0 & 1,0 & 0,0 & 1,0 & 416,30 & 420,49 \\
\hline Primer grado & 0,06 & 0,07 & 0,23 & 0,26 & 0,0 & 1,0 & 0,0 & 1,0 & 297,87 & 332,74 \\
\hline Segundo grado & 0,10 & 0,10 & 0,30 & 0,30 & 0,0 & 1,0 & 0,0 & 1,0 & 328,19 & 331,88 \\
\hline Tercer grado & 0,18 & 0,17 & 0,39 & 0,38 & 0,0 & 1,0 & 0,0 & 1,0 & 368,54 & 374,27 \\
\hline Cuarto grado & 0,59 & 0,59 & 0,49 & 0,49 & 0,0 & 1,0 & 0,0 & 1,0 & 457,92 & 463,50 \\
\hline Quinto grado & 0,07 & 0,07 & 0,26 & 0,25 & 0,0 & 1,0 & 0,0 & 1,0 & 488,76 & 484,61 \\
\hline Retraso & 0,32 & 0,33 & 0,46 & 0,47 & 0,0 & 1,0 & 0,0 & 1,0 & 342,81 & 350,74 \\
\hline ISEC & $-0,35$ & $-0,51$ & 1,05 & 1,18 & $-3,7$ & 2,4 & $-4,3$ & 2,8 & & \\
\hline
\end{tabular}

Variables centro

\begin{tabular}{|c|c|c|c|c|c|c|c|c|c|}
\hline Efecto par (ISEC) & $-0,35$ & $-0,51$ & 0,63 & 0,77 & $-2,3$ & 1,3 & $-2,7$ & 1,6 & \\
\hline Tamaño colegio & 531,12 & 435,16 & 335,86 & 248,90 & 9,0 & 2535,0 & 30,0 & 1275,0 & \\
\hline Alumnos por profesor & 17,79 & 15,86 & 9,43 & 5,53 & 1,9 & 65,0 & 2,0 & 29,6 & \\
\hline Escasez de materiales educativos & 2,86 & 2,53 & 1,02 & 1,07 & 1,0 & 4,0 & 1,0 & 4,0 & \\
\hline Escasez de profesores de matemática & 2,43 & 1,89 & 1,04 & 1,04 & 1,0 & 4,0 & 1,0 & 4,0 & \\
\hline Porcentaje de profesores titulados & 0,53 & 0,60 & 0,21 & 0,19 & 0,0 & 1,0 & 0,1 & 1,0 & \\
\hline Montevideo y área metropolitana & 0,48 & 0,46 & 0,50 & 0,50 & 0,0 & 1,0 & 0,0 & 1,0 & $440,64 \quad 443,31$ \\
\hline Ciudades capitales del interior & 0,32 & 0,32 & 0,47 & 0,47 & 0,0 & 1,0 & 0,0 & 1,0 & $412,65 \quad 412,72$ \\
\hline Ciudades menores del interior & 0,11 & 0,13 & 0,31 & 0,33 & 0,0 & 1,0 & 0,0 & 1,0 & $406,87 \quad 423,77$ \\
\hline Localidades menos de 5.000 habitantes & 0,09 & 0,09 & 0,29 & 0,29 & 0,0 & 1,0 & 0,0 & 1,0 & $376,85396,50$ \\
\hline \multicolumn{10}{|l|}{ Variables institucionales } \\
\hline Selectividad & 0,10 & 0,09 & 0,31 & 0,29 & 0,0 & 1,0 & 0,0 & 1,0 & $470,00 \quad 442,64$ \\
\hline Institución pública & 0,86 & 0,85 & 0,35 & 0,36 & 0,0 & 1,0 & 0,0 & 1,0 & $409,24 \quad 414,85$ \\
\hline Institución privada & 0,14 & 0,15 & 0,35 & 0,36 & 0,0 & 1,0 & 0,0 & 1,0 & $501,24 \quad 495,21$ \\
\hline
\end{tabular}

Fuente: elaboración propia sobre la base de datos de la Organización de Cooperación y Desarrollo Económicos (ocDE), "PISA 2003" y "PISA 2006" [en línea] http://www.pisa.oecd.org/document/51/0,3746,en_32252351_32235731_39732595_1_1_1_1,00.html

Nota: valores expandidos para toda la población.

ISEC: índice socioeconómico y cultural.

\begin{tabular}{|c|c|c|c|}
\hline & \multirow{2}{*}{ Efecto total promedio } & \multicolumn{2}{|c|}{ Cambios solo en: } \\
\hline & & Públicos & Privados \\
\hline $\begin{array}{l}\text { PISA Matemática } 2003 \\
\text { PISA Matemática } 2006\end{array}$ & $\begin{array}{l}422,20 \\
426,80\end{array}$ & & \\
\hline Diferencia total PISA Matemática & 4,60 & & \\
\hline $\begin{array}{l}\text { Efecto características } \\
\text { Efecto peso } \\
\text { Efecto características+peso } \\
\text { Efecto precio } \\
\text { Efecto características+peso+precio } \\
\text { Efecto choice } \\
\text { Efecto características+peso+precio+choice } \\
\text { Efecto residuos }\end{array}$ & $\begin{array}{r}9,24 \\
-2,76 \\
-2,11 \\
7,69 \\
7,59 \\
-0,05 \\
5,91 \\
0,00\end{array}$ & $\begin{array}{r}8,45 \\
-3,99 \\
-4,25 \\
8,83 \\
6,67 \\
-0,05 \\
6,52 \\
0,00\end{array}$ & $\begin{array}{r}0,80 \\
1,22 \\
2,17 \\
-1,14 \\
1,08 \\
-0,05 \\
-0,90 \\
0,00\end{array}$ \\
\hline Efecto características+peso+precio+choice+residuos & 5,93 & 6,58 & $-0,93$ \\
\hline
\end{tabular}

Fuente: elaboración propia sobre la base de datos de la Organización de Cooperación y Desarrollo Económicos (ocDE), “PISA 2003” y “PISA 2006" [en línea] http://www.pisa.oecd.org/document/51/0,3746,en_32252351_32235731_39732595_1_1_1_1,00.html

Nota: valores expandidos para toda la población. 
CUADRO A.4

Resultados de las microsimulaciones de características por decil de puntaje, 2003-2006

\begin{tabular}{|c|c|c|c|c|c|c|}
\hline & \multicolumn{4}{|c|}{ Efecto total y por decil (X) } & \multicolumn{2}{|c|}{ Cambios solo en: } \\
\hline & Total & 2 & 5 & 9 & Públicos & Privados \\
\hline PISA Matemática 2003 & 422,20 & 318,07 & 410,99 & 523,22 & & \\
\hline PISA Matemática 2006 & 426,80 & 325,26 & 417,46 & 523,09 & & \\
\hline Diferencia total PISA Matemática & 4,60 & 7,19 & 6,47 & $-0,13$ & & \\
\hline Variables estudiante & 7,05 & 17,05 & 8,17 & $-1,18$ & 6,85 & 0,20 \\
\hline Sexo $($ mujer $=1)$ & $-1,82$ & 2,23 & $-2,99$ & $-4,05$ & $-1,84$ & 0,03 \\
\hline Tercer grado & $-1,99$ & $-4,79$ & $-2,46$ & $-0,13$ & $-2,13$ & 0,14 \\
\hline Cuarto grado & 11,94 & 7,00 & 7,92 & 18,74 & 11,60 & 0,34 \\
\hline Quinto grado & $-1,25$ & $-1,05$ & $-2,19$ & $-1,46$ & $-0,76$ & $-0,49$ \\
\hline Retraso & 0,33 & 1,95 & 0,06 & $-0,61$ & 0,33 & 0,00 \\
\hline ISEC & $-0,15$ & $-0,83$ & $-0,34$ & 0,57 & $-0,34$ & 0,19 \\
\hline Variables centro & 2,29 & $-0,32$ & 2,25 & 4,74 & 1,67 & 0,62 \\
\hline Efecto par (ISEC) & 0,25 & $-1,20$ & 0,01 & 1,72 & $-0,28$ & 0,53 \\
\hline Tamaño del colegio & $-0,02$ & 0,01 & $-0,10$ & $-0,04$ & $-0,01$ & $-0,01$ \\
\hline Alumnos por profesor & 0,16 & 0,11 & 0,12 & 0,27 & 0,13 & 0,03 \\
\hline Escasez de materiales educativos & 1,00 & 0,18 & 0,75 & 1,78 & 0,76 & 0,24 \\
\hline Escasez de profesores matemática & $-0,91$ & $-0,76$ & $-0,98$ & $-0,98$ & $-0,87$ & $-0,04$ \\
\hline Porcentaje de profesores titulados & 1,63 & 1,98 & 1,64 & 1,39 & 1,70 & $-0,06$ \\
\hline Montevideo y área metropolitana & 0,26 & $-0,42$ & 0,42 & 1,00 & 0,26 & 0,00 \\
\hline Rural & $-0,08$ & $-0,14$ & $-0,06$ & 0,27 & $-0,01$ & $-0,07$ \\
\hline Variables institucionales & $-0,09$ & 0,36 & $-0,25$ & 0,14 & $-0,07$ & $-0,02$ \\
\hline Selectividad & $-0,09$ & 0,36 & $-0,25$ & 0,14 & $-0,07$ & $-0,02$ \\
\hline Variables estudiante y centro & 9,34 & 17,87 & 10,71 & 2,82 & 8,52 & 0,82 \\
\hline Variables estudiante e institucionales & 6,96 & 17,04 & 8,16 & $-1,32$ & 6,78 & 0,18 \\
\hline Variables centro e institucionales & 2,19 & 0,03 & 1,89 & 4,51 & 1,59 & 0,60 \\
\hline Todas las variables & 9,24 & 17,87 & 10,77 & 2,46 & 8,45 & 0,80 \\
\hline
\end{tabular}

Fuente: elaboración propia sobre la base de datos de la Organización de Cooperación y Desarrollo Económicos (OCDE), "PISA 2003” y “PISA 2006” [en línea] http://www.pisa.oecd.org/document/51/0,3746,en_32252351_32235731_39732595_1_1_1_1,00.html

Nota: valores expandidos para toda la población.

ISEC: índice socioeconómico y cultural.

\begin{tabular}{|c|c|c|c|c|c|c|}
\hline & \multicolumn{4}{|c|}{ Efecto total y por decil $(\beta)$} & \multicolumn{2}{|c|}{ Cambios solo en: } \\
\hline & Total & 2 & 5 & 9 & Públicos & Privados \\
\hline PISA Matemática 2003 & 422,20 & 318,07 & 410,99 & 523,25 & & \\
\hline PISA Matemática 2006 & 426,80 & 325,26 & 417,46 & 523,09 & & \\
\hline Diferencia total PISA Matemática & 4,60 & 7,19 & 6,47 & $-0,16$ & & \\
\hline Variables estudiante & $-36,54$ & $-29,29$ & $-35,38$ & $-43,09$ & $-22,63$ & $-13,91$ \\
\hline Sexo $($ mujer $=1)$ & $-2,80$ & $-3,33$ & $-3,17$ & $-2,09$ & $-2,85$ & 0,04 \\
\hline Tercer grado & $-2,41$ & $-3,67$ & $-2,57$ & $-1,60$ & $-1,22$ & $-1,19$ \\
\hline Cuarto grado & $-22,50$ & $-11,91$ & $-22,54$ & $-32,44$ & $-10,81$ & $-11,69$ \\
\hline Quinto grado & $-3,25$ & $-1,04$ & $-3,36$ & $-5,61$ & $-1,99$ & $-1,26$ \\
\hline
\end{tabular}




\begin{tabular}{|c|c|c|c|c|c|c|}
\hline & \multicolumn{4}{|c|}{ Efecto total y por decil $(\beta)$} & \multicolumn{2}{|c|}{ Cambios solo en: } \\
\hline & Total & 2 & 5 & 9 & Públicos & Privados \\
\hline Retraso & $-5,05$ & $-11,62$ & $-4,31$ & $-0,47$ & $-4,89$ & $-0,16$ \\
\hline ISEC & $-0,52$ & $-1,55$ & $-0,88$ & 0,66 & $-0,87$ & 0,34 \\
\hline Variables centro & $-8,87$ & $-6,77$ & $-8,65$ & $-10,89$ & $-6,31$ & $-2,56$ \\
\hline Efecto par (ISEC) & 1,29 & $-0,84$ & 0,39 & 3,80 & $-0,58$ & 1,87 \\
\hline Tamaño del colegio & $-4,25$ & $-3,73$ & $-4,65$ & $-4,40$ & $-4,24$ & $-0,01$ \\
\hline Alumnos por profesor & 0,61 & $-0,42$ & 0,08 & 1,96 & $-0,51$ & 1,12 \\
\hline Escasez de materiales educativos & 3,27 & 2,47 & 3,00 & 4,09 & 2,15 & 1,11 \\
\hline Escasez de profesores de matemática & $-5,32$ & $-5,10$ & $-5,32$ & $-5,60$ & $-4,64$ & $-0,68$ \\
\hline Porcentaje de profesores titulados & $-6,45$ & $-1,50$ & $-4,54$ & $-11,42$ & 0,34 & $-6,79$ \\
\hline Montevideo y área metropolitana & 1,67 & 0,79 & 1,30 & 2,55 & 0,77 & 0,90 \\
\hline Rural & 0,30 & 0,71 & 0,24 & 0,18 & 0,38 & $-0,07$ \\
\hline Variables institucionales & $-0,26$ & $-0,28$ & $-0,37$ & $-0,09$ & $-0,39$ & 0,13 \\
\hline Selectividad & $-0,26$ & $-0,28$ & $-0,37$ & $-0,09$ & $-0,39$ & 0,13 \\
\hline Constante & 53,36 & 45,28 & 48,93 & 64,49 & 38,16 & 15,20 \\
\hline Variables estudiante y centro & $-45,67$ & $-37,30$ & $-44,88$ & $-53,33$ & $-29,33$ & $-16,34$ \\
\hline Variables estudiante e institucionales & $-36,80$ & $-29,48$ & $-35,74$ & $-43,34$ & $-23,02$ & $-13,78$ \\
\hline Variables centro e institucionales & $-9,13$ & $-7,01$ & $-8,99$ & $-11,00$ & $-6,70$ & $-2,43$ \\
\hline Todas las variables & $-45,67$ & $-37,30$ & $-44,88$ & $-53,33$ & $-29,33$ & $-16,34$ \\
\hline Variables y constante & 7,69 & 12,15 & 7,44 & 3,90 & 8,83 & $-1,14$ \\
\hline
\end{tabular}

Fuente: elaboración propia sobre la base de datos de la Organización de Cooperación y Desarrollo Económicos (OCDE), "PISA 2003” y "PISA 2006" [en línea] http://www.pisa.oecd.org/document/51/0,3746,en_32252351_32235731_39732595_1_1_1_1,00.html

Nota: valores expandidos para toda la población.

ISEC: índice socioeconómico y cultural.

CUADRO A.6

Efecto de las microsimulaciones sobre los indicadores de distribución

\begin{tabular}{|c|c|c|c|c|c|c|c|c|c|}
\hline & \multicolumn{3}{|c|}{$\begin{array}{l}\text { Cambios en un solo tipo } \\
\text { de colegio }\end{array}$} & \multicolumn{3}{|c|}{$\begin{array}{l}\text { Cambios en un solo } \\
\text { tipo de colegio }\end{array}$} & \multicolumn{3}{|c|}{$\begin{array}{l}\text { Cambios en un solo } \\
\text { tipo de colegio }\end{array}$} \\
\hline & Gini $^{\mathrm{a}}$ & Públicos & Privados & Theil $^{\mathrm{b}}$ & Públicos & Privados & Entropía & Públicos & Privados \\
\hline Diferencia total PISA Matemática & $-0,004$ & $-0,003$ & 0,001 & $-0,001$ & $-0,001$ & 0,000 & $-0,002$ & $-0,001$ & 0,000 \\
\hline Efecto características & $-0,009$ & $-0,009$ & 0,039 & $-0,003$ & $-0,003$ & 0,014 & $-0,003$ & $-0,003$ & 0,013 \\
\hline Efecto peso & 0,003 & 0,004 & 0,039 & 0,001 & 0,002 & 0,013 & 0,001 & 0,002 & 0,013 \\
\hline Efecto características+peso & $-0,001$ & $-0,001$ & 0,039 & 0,000 & 0,000 & 0,014 & 0,000 & 0,000 & 0,013 \\
\hline Efecto precio & $-0,007$ & $-0,005$ & 0,038 & $-0,003$ & $-0,002$ & 0,013 & $-0,003$ & $-0,002$ & 0,013 \\
\hline Efecto características+peso+precio & $-0,010$ & $-0,009$ & 0,039 & $-0,004$ & $-0,003$ & 0,013 & $-0,003$ & $-0,003$ & 0,013 \\
\hline Efecto choice & 0,000 & 0,002 & 0,039 & 0,000 & 0,001 & 0,013 & 0,000 & 0,001 & 0,013 \\
\hline Efecto características+peso+precio+choice & $-0,005$ & $-0,005$ & 0,040 & $-0,002$ & $-0,002$ & 0,014 & $-0,002$ & $-0,002$ & 0,013 \\
\hline Efecto residuos & $-0,002$ & 0,000 & 0,038 & $-0,001$ & 0,000 & 0,013 & $-0,001$ & 0,000 & 0,013 \\
\hline $\begin{array}{l}\text { Efecto características+peso+precio+ } \\
\text { choice+residuos }\end{array}$ & $-0,008$ & $-0,007$ & 0,039 & $-0,003$ & $-0,002$ & 0,013 & $-0,003$ & $-0,002$ & 0,013 \\
\hline
\end{tabular}

Fuente: elaboración propia sobre la base de datos de la Organización de Cooperación y Desarrollo Económicos (OCDE), "PISA 2003” y “PISA 2006" [en línea] http://www.pisa.oecd.org/document/51/0,3746,en_32252351_32235731_39732595_1_1_1_1,00.html

Nota: El índice de entropía se calculó con beta $=2$.

Efecto choice: efecto selección de establecimiento.

a Coeficiente de Gini.

b Índice de Theil. 
Bibliografía

Almeida dos Reis, J. y R. Paes de Barros (1991), "Wage inequality and the distribution of education: a study of the evolution of regional differences in inequality in metropolitan Brazil", Journal of Development Economics, vol. 36, № 1, Amsterdam, Elsevier.

Amarante, V. e I. Perazzo (2008), "Crecimiento económico y pobreza en Uruguay. 1991-2006", Documento de trabajo, N $09 / 08$, Montevideo, Instituto de Economía, Facultad de Ciencias Económicas y de Administración, Universidad de la República.

Ammermüller, A. (2004), "PISA: what makes the difference? Explaining the gap in PISA test scores between Finland and Germany", Discussion Paper, № 04-04, Mannheim, Centre for European Economic Research.

ANEP (Administración Nacional de Educación Pública) (2007a), "Elementos para analizar la evolución reciente de la matrícula de educación secundaria", Montevideo.

(2007b), "Uruguay en PISA 2006. Primeros resultados en ciencias, matemática y lectura del Programa Internacional de Evaluación de Estudiantes", Montevideo.

(2004), "Primer informe nacional. PISA 2003 Uruguay", Montevideo.

Bellei, C. y otros (2009), "Qué explica las diferencias de resultados PISA Lectura entre Chile y algunos países de la OCDE y América Latina", ¿Qué nos dice PISA sobre la educación de los jóvenes en Chile? Nuevos análisis y perspectivas sobre los resultados en PISA 2006", Santiago de Chile, Ministerio de Educación, Unidad de Currículum y Evaluación.

Blinder, A. (1973), "Wage discrimination: reduced form and structural estimates", Journal of Human Resources, vol. 4, No 8, Wisconsin, The University of Wisconsin Press.

Bourguignon, F., M. Fournier y M. Gurgand (1998), "Distribution, development, and education in Taiwan, 1979-94", Documento de trabajo, París, DELTA.

Cardozo, S. (2008), "Políticas educativas, logros y desafíos del sector en Uruguay 1990-2008", Cuadernos de la ENIA, No 3, Montevideo, Comité de Coordinación Estratégica de Infancia y Adolescencia.

Fuchs, T. y L. Woessmann (2004), "What accounts for international differences in student performance? A re-examination using PISA data", IZA Discussion Papers, N 1287. Bonn, Institute for the Study of Labor.
Hanushek, E. y L. Woessmann (2010), "The economics of international differences in educational achievement", CESifo Working Paper, $\mathrm{N}^{\circ}$ 3037, Munich, CEsifo.

Juhn, C., K. Murphy y B. Pierce (1993), "Wage inequality and the rise in return to skill", Journal of Political Economy, vol. 101, $\mathrm{N}^{\circ} 3$, Chicago, University of Chicago Press.

Llambí, C. y M. Perera (2008), "La función de producción educativa: el posible sesgo en la estimación de efectos "institucionales" con los datos PISA. El caso de las escuelas de tiempo completo", Montevideo, Centro de Investigaciones Económicas (CINVE).

Méndez, N. y M. Zerpa (2009), "Desigualdad en las capacidades educativas en Uruguay y Chile", documento presentado en el I Seminario sobre economía de la educación, Montevideo, Facultad de Ciencias Económicas y Administración, Universidad de la República.

Oaxaca, R. (1973), "Male-female wage differentials in urban labor markets", International Economic Review, vol. 14, No 3, Filadelfia, University of Pennsylvania/Osaka University Institute of Social and Economic Research Association.

oCDE (Organización de Cooperación y Desarrollo Económicos) (2006), PISA 2003: Manual de análisis de datos. Usuarios de SPSS $®$, Madrid, Instituto Nacional de Evaluación y Calidad del Sistema Educativo (INECSE).

Pellegrino, A. y M. Koolhaas (2008), "Migración internacional: los hogares de los emigrantes", Demografía de una sociedad en transición: la población uruguaya a inicios del siglo XXI, C. Varela (coord.), Montevideo, Ediciones Trilce.

PNUD (Programa de las Naciones Unidas para el Desarrollo) (2008), Informe sobre desarrollo humano en Uruguay 2008. Política, políticas y desarrollo humano, Montevideo.

Valenzuela, J. y otros (2009a), "Causas que explican el mejoramiento de los resultados obtenidos por los estudiantes chilenos en PISA 2006 respecto a PISA 2001. Aprendizajes y políticas”, Santiago de Chile, Fondo de Investigación y Desarrollo en Educación, Ministerio de Educación.

(2009b), "Qué explica las diferencias de resultados PISA Matemática entre Chile y algunos países de la OCDE y América Latina”, ¿Qué nos dice PISA sobre la educación de los jóvenes en Chile? Nuevos análisis y perspectivas sobre los resultados en PISA 2006, Santiago de Chile, Ministerio de Educación, Unidad de Currículum y Evaluación. 